\title{
Deep low-frequency radio observations of the NOAO Boötes field
}

\section{Data reduction and catalog construction $\star$}

\author{
H. T. Intema ${ }^{1,2, \star \star}$, R. J. van Weeren ${ }^{1}$, H. J. A. Röttgering ${ }^{1}$, and D. V. Lal ${ }^{3,4}$ \\ Leiden Observatory, Leiden University, PO Box 9513, 2300 RA, Leiden, The Netherlands \\ 2 National Radio Astronomy Observatory, Charlottesville, VA, USA \\ e-mail: hintema@nrao.edu \\ ${ }^{3}$ National Centre for Radio Astrophysics, Univerity of Pune, India \\ ${ }^{4}$ Max-Planck-Institut für Radioastronomie, Bonn, Germany
}

Received 12 February 2010 / Accepted 23 September 2011

\begin{abstract}
In this article we present deep, high-resolution radio interferometric observations at $153 \mathrm{MHz}$ to complement the extensively studied NOAO Boötes field. We provide a description of the observations, data reduction and source catalog construction. From our single pointing GMRT observation of $\sim 12 \mathrm{~h}$ we obtain a high-resolution $\left(26^{\prime \prime} \times 22^{\prime \prime}\right)$ image of $\sim 11.3$ square degrees, fully covering the Boötes field region and beyond. The image has a central noise level of $\sim 1.0 \mathrm{mJy}^{\mathrm{beam}}{ }^{-1}$, which rises to $2.0-2.5 \mathrm{mJy}^{\mathrm{beam}}{ }^{-1}$ at the field edge, placing it amongst the deepest $\sim 150 \mathrm{MHz}$ surveys to date. The catalog of 598 extracted sources is estimated to be $\sim 92$ percent complete for $>10 \mathrm{mJy}$ sources, while the estimated contamination with false detections is $<1$ percent. The low rms position uncertainty of $1.24^{\prime \prime}$ facilitates accurate matching against catalogs at optical, infrared and other wavelengths. Differential source counts are determined down to $\lesssim 10 \mathrm{mJy}$. There is no evidence for flattening of the counts towards lower flux densities as observed in deep radio surveys at higher frequencies, suggesting that our catalog is dominated by the classical radio-loud AGN population that explains the counts at higher flux densities. Combination with available deep $1.4 \mathrm{GHz}$ observations yields an accurate determination of spectral indices for 417 sources down to the lowest $153 \mathrm{MHz}$ flux densities, of which 16 have ultra-steep spectra with spectral indices below -1.3 . We confirm that flattening of the median spectral index towards low flux densities also occurs at this frequency. The detection fraction of the radio sources in NIR $K_{\mathrm{S}}$-band is found to drop with radio spectral index, which is in agreement with the known correlation between spectral index and redshift for brighter radio sources.
\end{abstract}

Key words. surveys - galaxies: active - radio continuum: galaxies

\section{Introduction}

Surveying the radio sky at low frequencies $(\$ 300 \mathrm{MHz})$ is a unique tool for investigating many questions related to the formation and evolution of massive galaxies, quasars and clusters of galaxies (e.g., Miley \& De Breuck 2008). Low-frequency radio observations benefit from the steepness of radio spectra of various types of cosmic radio sources, such as massive HzRGs (high-redshift radio galaxies; redshift $z \gtrsim 2$ ) and diffuse halo \& relic emission in nearby galaxy clusters $(z \lesssim 0.1)$.

HzRGs are amongst the most massive galaxies in the early Universe (e.g., Miley \& De Breuck 2008), usually located in forming galaxy clusters with total masses of more than $10^{14} M_{\odot}$ (e.g., Venemans et al. 2007). The most efficient way of finding HzRGs is to focus on USS (ultra-steep spectrum; $S_{v} \propto v^{\alpha}$ with $\alpha \lesssim-1$ ) radio sources (Röttgering et al. 1997; De Breuck et al. 2002). This was reinforced by Klamer et al. (2006) who showed that the radio spectra of HzRGs in general do not show spectral curvature, but are straight. The USS selection criteria appear to hold down to very low flux levels (e.g., Afonso et al. 2011).

* Full Table 3 is only available at the CDS via anonymous ftp to cdsarc.u-strasbg.fr $(130.79 .128 .5)$ or via

http://cdsarc.u-strasbg.fr/viz-bin/qcat?]/A+A/535/A38

$\star \star$ Jansky Fellow of the National Radio Astronomy Observatory.
Concentrating on the faintest sources from surveys made at the lowest frequencies is therefore an obvious way of pushing the distance limit for HzRGs beyond the present highest redshift of TN J0924-2201 at $z=5.1$ (van Breugel et al. 1999) and probing massive galaxy formation into the epoch of reionization.

Galaxy clusters containing diffuse radio sources appear to have large X-ray luminosities and galaxy velocity dispersions (e.g., Hanisch 1982), which are thought to be characteristics of cluster merger activity (e.g., Giovannini \& Feretti 2000; Kempner \& Sarazin 2001). Synchrotron halos and relics provide unique diagnostics for studying the magnetic field, plasma distribution and gas motions within clusters, important inputs to models of cluster evolution (e.g., Feretti \& Johnston-Hollitt 2004). Cluster synchrotron emission is known to be related to the X-ray gas and pinpoints shocks in the gas. Further, cluster radio emission usually has steep radio spectra $(\alpha<-1)$, the radiating electrons are old and can provide fossil records of the cluster history (e.g., Miley 1980).

Several low-frequency surveys have been performed in the past, such as the Cambridge surveys $3 \mathrm{C}, 4 \mathrm{C}, 6 \mathrm{C}$ and $7 \mathrm{C}$ at 159 , 178, 151 and again $151 \mathrm{MHz}$, respectively (Edge et al. 1959; Bennett 1962; Pilkington \& Scott 1965; Gower et al. 1967; Hales et al. 1988, 2007), the UTR-2 sky survey between $10-25 \mathrm{MHz}$ (Braude et al. 2002), the VLSS at $74 \mathrm{MHz}$ (Cohen et al. 2007) 
and the ongoing MRT sky survey at $151.5 \mathrm{MHz}$ (e.g., Pandey $\&$ Shankar 2007). These surveys are limited in sensitivity and angular resolution, mainly due to man-made RFI, ionospheric phase distortions and wide-field imaging problems. Recent developments in data reduction techniques make it possible to perform deeper surveys $\left(\$ 50 \mathrm{mJy}^{\text {beam }}{ }^{-1}\right)$ of the low-frequency sky at higher resolution $\left(\$ 30^{\prime \prime}\right)$. One telescope that significantly improved this situation is the GMRT. A few deep, single-pointing surveys at $153 \mathrm{MHz}$ (e.g., Ishwara-Chandra \& Marathe 2007; Sirothia et al. 2009; Ishwara-Chandra et al. 2010) have been performed, yielding noise levels between $0.7-2 \mathrm{mJy} \mathrm{beam}^{-1}$ and resolutions between 20-30". And the $\mathrm{TGSS}^{1}$ is a new, ongoing $153 \mathrm{MHz}$ GMRT sky survey, aimed at covering the full northern sky down to Dec $>-30^{\circ}$ at a $\sim 20^{\prime \prime}$ resolution and a 7-9 $\mathrm{mJy}_{\text {beam }}^{-1}$ noise.

In this article we present deep, high-resolution GMRT observations at $153 \mathrm{MHz}$ of the NOAO Boötes field. The Boötes field is a large ( $\sim 9$ square degree) northern field that has been targeted by surveys spanning the entire electromagnetic spectrum. This field has been extensively surveyed with radio telescopes including the WSRT at $1.4 \mathrm{GHz}$ (de Vries et al. 2002), the VLA at $325 \mathrm{MHz}$ (Croft et al. 2008), and will be complemented with deep $74 \mathrm{MHz}$ EVLA observations. The large northern NDWFS survey (Jannuzi \& Dey 1999) provided 6 colour images $\left(B_{W} R I J H K\right)$ to very faint optical and NIR flux limits. Additional, deeper NIR images in $J K_{\mathrm{S}}$ are available from the FLAMEX survey (Elston et al. 2006), while additional z-band images are available from the $z$ Bootes campaign (Cool 2007). The entire area has also been surveyed by Spitzer in seven IR bands ranging from 3.6 to $160 \mu \mathrm{m}$ (Eisenhardt et al. 2004; Houck et al. 2005). Chandra has covered this area in the energy range of $0.5-7 \mathrm{keV}$ to a depth of $\sim 10^{-14} \mathrm{ergs} \mathrm{s}^{-1} \mathrm{~cm}^{-2}$, yielding 3200 quasars and 30 luminous X-ray clusters up to redshift $z \sim 1$ (Murray et al. 2005; Kenter et al. 2005). The UV space telescope GALEX has covered the Boötes field. All the 10000 galaxies brighter than $R<19.2$ and X-ray/IRAC/MIPS QSOs brighter than $R<21.5$ have redshifts through the AGES project (Kochanek et al., in prep.). Based on the shallow Spitzer data, 3 HzRGs with photometric redshifts of $z>4$ have been identified in the Boötes field (Croft et al. 2008). Also, Cool et al. (2006) report the discovery of 3 quasars with spectroscopic redshifts $z>5$, while McGreer et al. (2006) found a quasar at $z=6.1$.

Given the size of the GMRT FoV (field-of-view 3.5 degrees) and angular resolution $\left(\sim 25^{\prime \prime}\right)$ at $153 \mathrm{MHz}$, the Boötes field is a well-matched region for conducting a deep survey. Combined with the existing multi-wavelength surveys, our deep $153 \mathrm{MHz}$ Boötes field observations allow for a complete study of faint ( $\gtrsim 20 \mathrm{mJy}$ ) low-frequency radio sources. For the data reduction, we used the recently developed SPAM calibration software that solves for spatially variant ionospheric phase rotations (Intema et al. 2009). In our initial analysis of the $153 \mathrm{MHz}$ source catalog we focus on determining source counts down to the detection limit, and identifying steep-spectrum radio sources that are candidate HzRGs. A more extended analysis of our source catalog in combination with the other multi-wavelength catalogs is planned in a subsequent article.

In Sect. 2, we describe the GMRT $153 \mathrm{MHz}$ observations and data reduction. In Sect. 3, we present details on the source extraction and catalog construction. Section 4 contains the initial analysis and discussion of the source population. Conclusions

\footnotetext{
${ }^{1}$ http://tgss.ncra.tifr.res.in/
}

Table 1. Overview of GMRT observations on the Boötes field.

\begin{tabular}{lcc}
\hline \hline Date & June 3, 2005 & June 4, 2005 \\
\hline LST range & $12-20 \mathrm{~h}$ & $10-19 \mathrm{~h}$ \\
Local time range $^{a}$ & $20: 00-04: 00$ & $18: 00-03: 00$ \\
Time on target & $359 \mathrm{~min}$ & $397 \mathrm{~min}$ \\
Primary calibrator & $3 \mathrm{C} 286$ & $3 \mathrm{C} 286$ \\
Time on calibrator & $94 \mathrm{~min}$ & $108 \mathrm{~min}$ \\
\hline Integration time & \multicolumn{2}{c}{$16.8 \mathrm{~s}$} \\
Polarizations & RR, LL \\
No. of channels & \multicolumn{2}{c}{128} \\
Channel width & \multicolumn{2}{c}{$62.5 \mathrm{KHz}$} \\
Total bandwidth & $8.0 \mathrm{MHz}$ \\
Target RA,Dec & $14^{\mathrm{h}} 32^{\mathrm{m}} 05.75^{\mathrm{s}},+34^{\circ} 16^{\prime} 47.5^{\prime \prime}$ \\
\hline
\end{tabular}

Notes. ${ }^{(a)}$ Indian Standard Time (IST) $=$ Universal Time $(\mathrm{UT})+5: 30$.

are presented in Sect. 5. Throughout this article, source positions are given in epoch $\mathbf{J} 2000$ coordinates.

\section{Observations and data reduction}

In this section, we describe the observations and data reduction steps that led to the production of the $153 \mathrm{MHz}$ image that is the basis of the survey.

\subsection{Observations}

We used the GMRT (e.g., Nityananda 2003) at $153 \mathrm{MHz}$ to observe the Boötes field, with the observing details given in Table 1 . There were typically 27 out of 30 antennas available during each observing run. We used 3C 286 as flux, bandpass and phase calibrator. Observation cycles of $\sim 50$ min were split into $\sim 38$ min on the target field and $\sim 10$ min scans on the calibrator. The relatively high overhead in calibrator observations was justified by the need to monitor the GMRT system stability, RFI conditions and ionospheric conditions, and to ensure consistency of the flux scale over time.

\subsection{Data reduction}

The data reduction was performed in two stages. The first stage consisted of "traditional" calibration, in which the flux scale, bandpass shapes and phase offsets were determined from the calibrator observations, transferred to the target field data, after which the target field was self-calibrated and imaged for several iterations. During the second stage of the data reduction, we made use of the recent SPAM software package (Intema et al. 2009) that incorporates direction-dependent ionospheric phase calibration.

For the traditional calibration we used the Astronomical Image Processing Sofware (AIPS; e.g., Bridle \& Greisen 1994) package. Initial flagging of bad baselines and excessive visibility amplitudes (mostly RFI) resulted in a $6.75 \mathrm{MHz}$ effective bandwidth. This was followed by amplitude, phase and bandpass calibration on 3 C 286. We adopted a Stokes I flux density of $31.01 \mathrm{Jy}$ on the Perley-Taylor $1999.2 \mathrm{scale}^{2}$, which is derived from the Baars et al. (1977) scale for 3C 295 (the flux scale is discussed in more detail in Sect. 3.3). To reduce the data volume, each 4 consecutive frequency channels were combined to form 27 channels of $0.25 \mathrm{MHz}$ each. After initial imaging of the target field (see Table 2), the calibration on the target field data

${ }^{2}$ Defined in the VLA Calibrator Manual; http://www.vla.nrao. edu/astro/calib/manual/baars.html 
Table 2. Overview of wide-field imaging parameters (upper panel) and SPAM parameters (lower panel).

\begin{tabular}{|c|c|}
\hline Field diameter ${ }^{a}$ & $6.8^{\circ}$ \\
\hline Pixel size & $4^{\prime \prime}$ \\
\hline Weighting $^{b}$ & robust 0.5 \\
\hline Wide-field imaging ${ }^{c, d}$ & $\begin{array}{l}\text { polyhedron (facet-based), } \\
\text { multi-frequency synthesis }\end{array}$ \\
\hline Number of facets & 199 \\
\hline Facet diameter & $0.57^{\circ}$ \\
\hline Facet separation & $0.47^{\circ}$ \\
\hline Deconvolution $^{e}$ & Cotton-Schwab CLEAN \\
\hline CLEAN box threshold & $5 \sigma$ \\
\hline CLEAN depth & $2 \sigma$ \\
\hline Restoring beam & $26^{\prime \prime} \times 22^{\prime \prime}\left(\mathrm{PA} 78^{\circ}\right)$ \\
\hline SPAM $^{f}$ calibration cycles & 2 \\
\hline Peeled sources ${ }^{g}$ & 24 \\
\hline \multirow[t]{3}{*}{ Layer heights (and weights) } & $100 \mathrm{~km}(0.25)$ \\
\hline & $200 \mathrm{~km}(0.50)$ \\
\hline & 400 km (0.25) \\
\hline Parameter $\gamma\left({ }^{h}\right)$ & $5 / 3$ \\
\hline Model parameters & 20 \\
\hline Model fit phase rms ${ }^{g}$ & $19.2^{\circ} \pm 3.0^{\circ}$ \\
\hline Peeling corrections applied & yes \\
\hline Reference catalog ${ }^{i}$ & NVSS \\
\hline
\end{tabular}

Notes. ${ }^{(a)}$ We map more than twice the HPBW diameter to allow for deconvolution of nearby bright sources. ${ }^{(b)}$ Briggs (1995). (c) Perley (1989); Cornwell \& Perley (1992). ${ }^{(d)}$ Conway et al. (1990). ${ }^{(e)}$ Schwab (1984); Cotton (1999); Cornwell et al. (1999). ${ }^{(f)}$ Intema et al. (2009) ${ }^{(g)}$ Specified for the final (second) calibration cycle only. ${ }^{(h)}$ Power-law slope of the assumed phase structure function. ${ }^{(i)}$ Condon et al. (1994, 1998).

was improved by three rounds of phase-only self-calibration and one round of amplitude \& phase self-calibration. To remove excessive visibilities at a lower level, the obtained source model was temporarily subtracted from the visibilities, after which the residual visibilities were manually and semi-automatically flagged per baseline. After re-imaging, the noise (rms of the image background) in the inner half of the (uncorrected) primary beam area, was approximately $1.4 \mathrm{mJy} \mathrm{beam}^{-1}$. Near the brightest three sources (with apparent flux densities larger than $1 \mathrm{Jy}$ ), the local noise was measured to be $\gtrsim 2.2 \mathrm{mJy}_{\text {beam }}^{-1}$.

Despite the good overall quality of the self-calibrated image from the traditional calibration, there were significant artifacts present in the background near bright sources, limiting the local dynamic range to a few hundred. To suppress artifacts related to direction-dependent calibration errors, we applied the SPAM algorithm on the self-calibrated(!) visibility data. The used SPAM parameters are reported in Table 2 (for details see Intema et al. 2009). This unconventional combination of self-calibration and SPAM is used to bypass the problem of antenna-based phase discontinuities, as observed in the calibration solutions for several antennas. Applying SPAM after self-calibration can be considered as a perturbation to the overall gradient correction found by self-calibration. The resulting noise in the inner half of the (uncorrected) primary beam area is on average $1.0 \mathrm{mJy}^{\text {beam }}{ }^{-1}$. The local noise near the brightest three sources is reduced to $\$ 2.0$ mJy beam $^{-1}$.

For an observation of $\sim 10 \mathrm{~h}$, the theoretical thermal noise level ${ }^{3}$ for the GMRT at $153 \mathrm{MHz}$ is estimated to be

\footnotetext{
3 Derived using the GMRT User Manual; http://gmrt.ncra. tifr.res.in/gmrt_hpage/Users/doc/manual/UsersManual/ index.html
}

$\sim 0.2-0.3 \mathrm{mJy}$ beam $^{-1}$. For our observation, the measured noise of $\sim 1.0 \mathrm{mJy}^{\text {beam }}{ }^{-1}$ in the central part of the field is a factor of 3 to 5 larger. In apparently source-free parts of the outer imaged area this drops to $0.75 \mathrm{mJy} \mathrm{beam}^{-1}$, indicating that residual sidelobe structure has a noticable contribution to the central noise. Although significantly improved as compared to the self-calibrated case, the residual sidelobe structure near the three bright sources, all located near the half-power radius of the primary beam area, limits the local dynamic range to $\sim 600$. We expect that the residual sidelobes are caused by directiondependent visibility amplitude errors due to pointing errors and non-circular primary beam patterns (e.g., Bhatnagar et al. 2008).

Another suspect for adding to the overall noise is residual RFI (see also Sect. 3.3). Especially on the most affected, short baselines, it can be difficult to distinguish between persistent broad-band RFI and true sky signal. In our approach we have attempted to save as many short baselines as possible, to preserve the sensitivity to large-scale emission. This could explain why our central noise level of $\sim 1.0 \mathrm{mJy}_{\text {beam }}{ }^{-1}$ is slightly higher than the quoted $0.7 \mathrm{mJy}_{\text {beam }}{ }^{-1}$ found by Sirothia et al. (2009); Ishwara-Chandra et al. (2010) for similar deep fields. In general, we find that a straightforward comparison of noise levels is non-trivial, as it depends on the flagging \& data selection strategy, the visibility weighting scheme used during imaging, and on how and where in the image the noise is measured.

\section{Catalog construction}

In this section we describe the construction of the source cata$\log$ that was extracted from the $153 \mathrm{MHz}$ image of the Boötes field. The image after SPAM calibration was scaled down by 4 percent to incorporate a correction to the flux calibrator scale (see Sect. 3.3). Next, the image was corrected for primary beam attenuation with a circular beam model $^{4}$ out to a 1.9 degree radius (out to 0.3 times the central beam response, slightly beyond the half-power radius), yielding a survey area of 11.3 square degrees. Figure 1 shows a map of the local noise of the circular survey area, which has a central area with a noise level of $\sim 1.0 \mathrm{mJy} \mathrm{beam}^{-1}$, a global increase of the local noise to 2.0 $2.5 \mathrm{mJy} \mathrm{beam}^{-1}$ when moving towards the field edge, and several small areas around bright sources where the local noise is approximately twice the surrounding noise level. The overall noise was found to be $1.7 \mathrm{mJy}_{\text {beam }}^{-1}$.

Although we used multi-frequency synthesis, the $0.25 \mathrm{MHz}$ width of individual frequency channels causes bandwidth smearing during imaging. Applying the standard formula (e.g., Thompson 1999) to our case, the radial broadening of sources at the edge of the field (at 1.9 degree from the pointing center) is estimated to be $\sim 11^{\prime \prime}$, which is half the minor axis of the restoring beam. Similarly, the visibility time resolution of $16.8 \mathrm{~s}$ causes time-average smearing in the order of $\sim 8^{\prime \prime}$ at the field's edge. This may appear problematic, but the majority of sources are detected within the inner part of the primary beam (both effects scale linearly with radial distance from the pointing center). The total flux density of smeared sources is conserved, but the peak flux drops. The effect of smearing on source extraction is discussed in Sect. 3.2.

\subsection{Source extraction}

We used the BDSM software package (Mohan 2008) to extract sources from our image. With our settings, BDSM estimates the

\footnotetext{
${ }^{4}$ See the GMRT User Manual.
} 


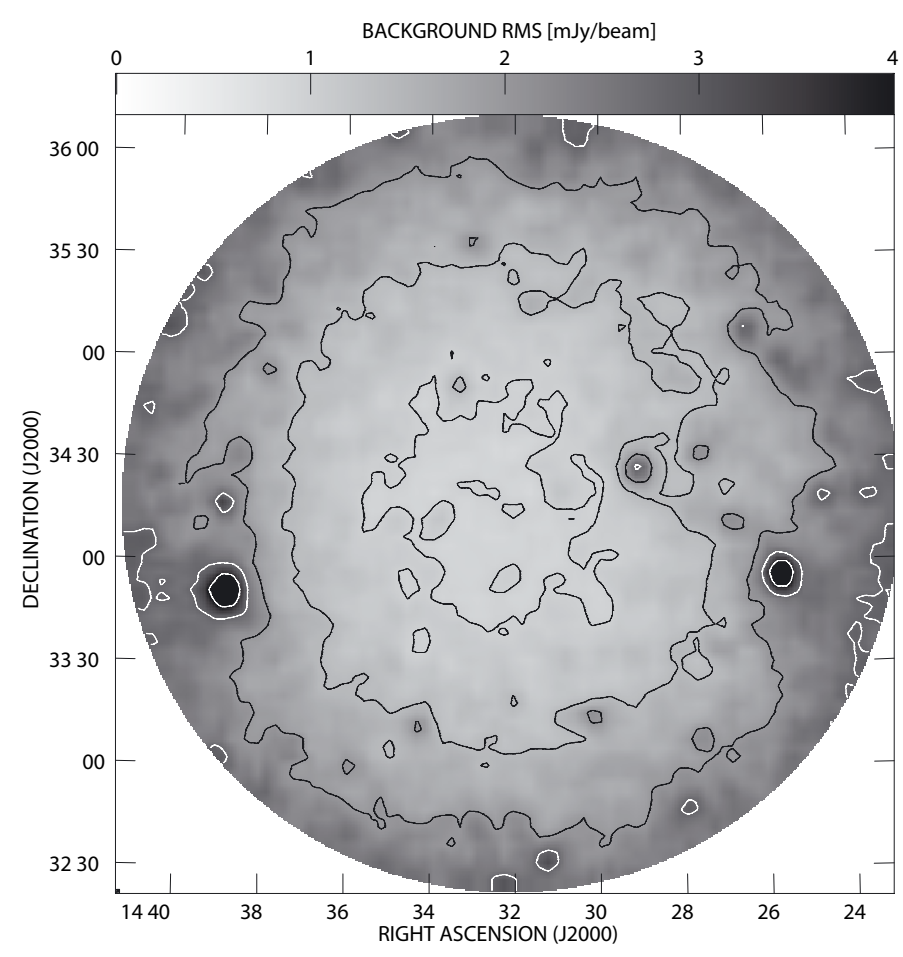

Fig. 1. The grayscale map represents the local noise as measured in the (primary beam corrected) Boötes field image. The overplotted contours mark lines of equal rms at $[1.0,1.4,2.0,2.8,4.0] \mathrm{mJy}^{-1}$ beam $^{-1}$ (white above $2.0 \mathrm{mJy}^{\text {beam }}{ }^{-1}$ ). The local enhancements in rms coincide with the positions of the brightest sources.

local background noise level $\sigma_{\mathrm{L}}$ over the map area, searches for pixels $>5 \sigma_{\mathrm{L}}$, expands the $5 \sigma_{\mathrm{L}}$ detections into islands by searching for adjacent pixels $>3 \sigma_{\mathrm{L}}$, rejecting islands smaller than 4 pixels. BDSM fits the emission in each island with one or multiple Gaussians, combines significantly overlapping Gaussians into sources, and determines the flux densities, shapes and positions of sources (including error estimates, following Condon 1997). This approach has led to very few false source detections (see Sect. 3.2).

BDSM detected 644 islands, for which the 935 fitted Gaussians were grouped into 696 distinct sources. Of these, 499 sources were fitted with a single Gaussian. Visual inspection of the image, complemented with a comparison against a very deep $1.4 \mathrm{GHz}$ map (see Sect. 3.3), resulted in the removal of 16 source detections in the near vicinity of six of the seven brightest sources. We also removed 4 sources that extended beyond the edge of the image. To facilitate total flux density measurements at the high flux end, we combined multiple source detections in single islands, and manually combined 50 additional source detections that were assigned to different islands but appeared to be associated in either the $153 \mathrm{MHz}$ image or the deep $1.4 \mathrm{GHz}$ map. The combined flux density is the sum of the individual components, while the combined position is a flux-weighted average (centroid). Error estimates of the positions and flux densities of the components are propagated into error estimates of the combined flux density and position. The final catalog consists of 598 sources.

Table 3 contains a sample of the full source catalog 5 . The source entries include the corrections discussed in the remainder of Sect. 3. The columns are: (1) source identifier, $(2,3)$ centroid position right ascension and declination, $(4,5)$ centroid

\footnotetext{
5 The full version of Table 3 is available electronically at the CDS.
}

position uncertainty along the right ascension and declination directions, $(6,7)$ integrated source flux and uncertainty, (8) local background rms noise level, and (9) number of Gaussians fitted to the source.

\subsection{Completeness and contamination}

We estimated the completeness of the $153 \mathrm{MHz}$ catalog by performing Monte-Carlo simulations. The source extraction process generated a residual image from which all detected source flux was subtracted. For our simulation, we inserted 1000 artificial point sources into the residual image, and used the same mechanism as described in Sect. 3.1 to extract them. The artificial source positions were selected randomly, but never within 50" of another source, a blanked region (near the image edge) or a $>10$ mJy residual. The source peak fluxes $S$ were chosen randomly within the range $3 \mathrm{mJy}$ to $3 \mathrm{Jy}$, while obeying the source count statistic $\mathrm{d} N / \mathrm{d} S \propto S^{-1.5}$, which produced statistically sufficient detection counts in all logarithmic flux bins. The simulation was repeated 20 times to improve the accuracy and to derive error estimates. The detection fraction as a function of peak flux is plotted in Fig. 2.

In this approach we have ignored several effects that may influence the detectability of sources, such as (i) the intrinsic size of sources, (ii) bandwidth- and time-average smearing, (iii) calibration errors, and (iv) imaging and deconvolution. The source detection algorithm uses a peak detection threshold. Sources that are resolved, smeared due to bandwidth- and time-averaging, or defocussed due to calibration errors will therefore have a decreased probability of detection. We have not attempted to model for the angular size distribution of sources at this frequency. However, previous observations show that the major fraction of low-frequency sources are unresolved at $\sim 25^{\prime \prime}$ resolution (e.g., Cohen et al. 2004; George \& Ishwara-Chandra 2009). In our catalog, more than 90 percent of the sources appear to have simple, near-Gaussian morphologies. Assuming the angular size distribution of sources changes slowly with source flux density, and assuming that smearing and defocussing affects sources of varying brightness in a statistically equal way, we can estimate the resolution bias from the catalog itself. For this purpose, we select a subset of 214 high $\mathrm{S} / \mathrm{N}$ sources with peak fluxes between 12 and $20 \sigma_{\mathrm{L}}$ and simple morphologies. The flux densities of these sources were scaled down by a factor of 4 to create an artificial population of sources near the detection threshold. After applying the $5 \sigma_{\mathrm{L}}$ detection criterium, we determined the detection fractions, both as a function of peak flux and total (integrated) flux density (Fig. 2). Although this approach suffers from low number statistics, the general trend of both detection fraction functions is similar but shifted upwards in flux by $\sim 15$ percent. We therefore assume that the total flux detection fraction is approximated by the peak flux detection fraction derived from the Monte-Carlo simulations, shifted upwards in flux by 15 percent.

To estimate the completeness of the catalog at various flux limits, the total flux detection fraction must be multiplied by the (normalized) true source flux distribution, and integrated from the flux limit upwards. The Euclidean-normalized differential source counts in Sect. 4.1 approximately follow a power-law over the larger part of the flux range of our sample, with a slope of 0.91 . The source flux distribution over the same flux range can therefore be approximated by a power-law with slope of $0.91-5 / 2=-1.59$. As it involves very few counts, the deviation of the power-law from the true source counts at the high flux end has little effect on our estimates, and is therefore ignored. Figure 2 shows the resulting completeness estimate as a 
H. T. Intema et al.: Deep low-frequency radio observations of the Boötes field. I.

Table 3. Sample of the full source catalog of GMRT $153 \mathrm{MHz}$ sources.

\begin{tabular}{|c|c|c|c|c|c|c|c|c|}
\hline (1) & $\begin{array}{l}\mathrm{RA} \\
{\left[{ }^{\circ}\right]} \\
(2)\end{array}$ & $\begin{array}{l}\text { Dec } \\
{\left[{ }^{\circ}\right]} \\
(3)\end{array}$ & $\begin{array}{l}\Delta \mathrm{RA} \\
{\left[{ }^{\prime \prime}\right]} \\
(4)\end{array}$ & $\begin{array}{l}\Delta \mathrm{Dec} \\
{\left[{ }^{\prime \prime}\right]} \\
(5)\end{array}$ & $\begin{array}{c}S_{153} \\
{[\mathrm{mJy}]} \\
(6)\end{array}$ & $\begin{array}{c}\Delta S_{153} \\
{[\mathrm{mJy}]} \\
(7)\end{array}$ & $\begin{array}{c}\text { Noise } \\
{\left[\mathrm{mJy} \mathrm{beam}^{-1}\right]} \\
(8)\end{array}$ & $N_{\text {Gauss }}$ \\
\hline $\mathrm{J} 142632+350815$ & 216.63367 & 35.13754 & 5.2 & 4.3 & 649.7 & 65.1 & 2.8 & 1 \\
\hline $\mathrm{J} 143810+340459$ & 219.54576 & 34.08315 & 3.9 & 2.8 & 641.2 & 64.2 & 2.0 & 1 \\
\hline $\mathrm{J} 143850+330225$ & 219.71068 & 33.04036 & 10.0 & 8.8 & 577.8 & 58.2 & 2.9 & 2 \\
\hline $\mathrm{J} 143134+351505$ & 217.89466 & 35.25165 & 5.2 & 3.5 & 567.8 & 56.9 & 1.6 & 2 \\
\hline $\mathrm{J} 142430+343630$ & 216.12544 & 34.60836 & 71.1 & 60.3 & 543.0 & 55.2 & 2.5 & 7 \\
\hline $\mathrm{J} 142445+341816$ & 216.19020 & 34.30447 & 7.2 & 5.3 & 507.1 & 50.9 & 2.9 & 1 \\
\hline $\mathrm{J} 143055+341933$ & 217.73287 & 34.32597 & 3.9 & 3.0 & 505.0 & 50.6 & 1.2 & 2 \\
\hline $\mathrm{J} 143256+353339$ & 218.23353 & 35.56104 & 4.7 & 4.0 & 503.8 & 50.5 & 2.0 & 1 \\
\hline $\mathrm{J} 143930+344628$ & 219.87580 & 34.77470 & 9.2 & 7.3 & 489.8 & 49.4 & 2.5 & 2 \\
\hline $\mathrm{J} 143128+355252$ & 217.86699 & 35.88132 & 11.6 & 11.6 & 459.4 & 46.5 & 2.3 & 3 \\
\hline
\end{tabular}

Notes. The columns are described in the text. An electronic version of the full table is available via CDS.
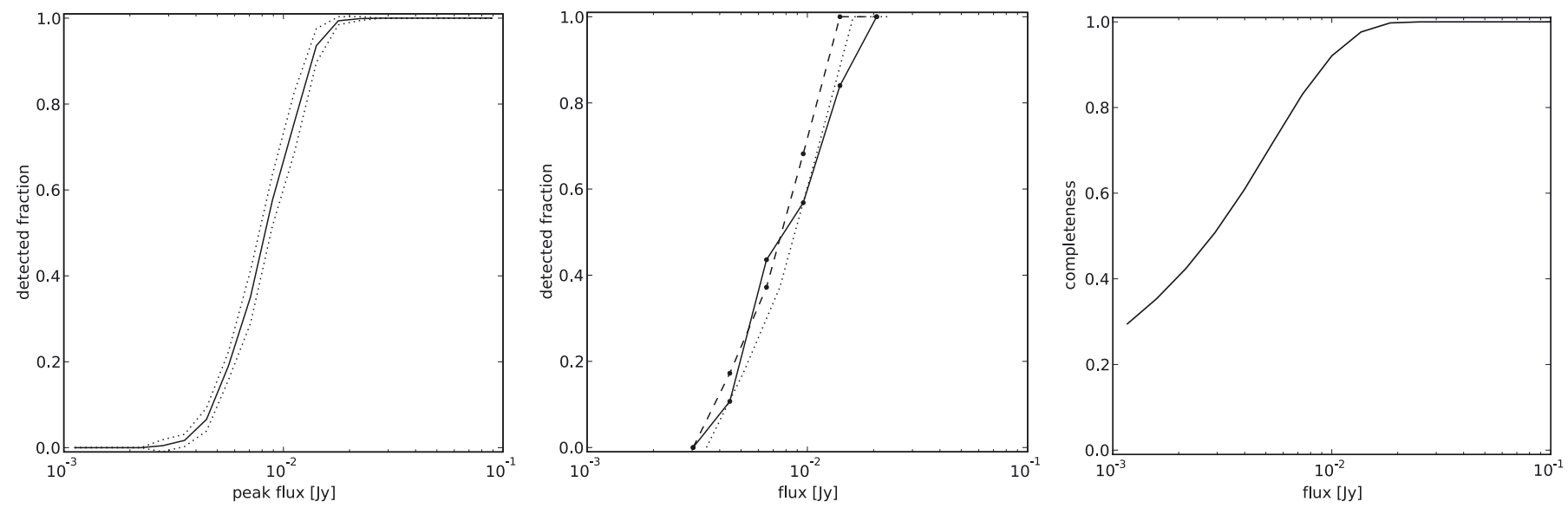

Fig. 2. Detection fractions and completeness as a function of flux. Left: result from 20 Monte-Carlo simulations, in which 1000 point sources with varying fluxes were inserted into the residual image, followed by a source extraction. The horizontal axis denotes the input (peak) flux. The solid line is the average detection fraction, and the dotted lines denote the $1 \sigma$ uncertainty. Middle: result from scaling the peak flux and total flux density of a subset of high $\mathrm{S} / \mathrm{N}$ sources with varying sizes down to the detection limit, and applying the $5 \sigma_{\mathrm{L}}$ detection criteria. The dashed line is the detection fraction as a function of peak flux, the solid line is the detection fraction as a function of total flux density, and the dotted line is the dashed line shifted in flux by 15 percent to approximately match the solid line. Right: estimated completeness of the extracted source catalog as a function of (total) flux limit.

function of total flux. From this plot, the estimated completeness is $\sim 70$ percent at $5.1 \mathrm{mJy}, \sim 92$ percent at $10 \mathrm{mJy}$ and $>99$ percent at $20 \mathrm{mJy}$.

A known effect that arises from the deconvolution process is CLEAN bias (e.g., Condon et al. 1994, 1998; Becker et al. 1995). This is a systematic negative offset in the recovered flux densities after deconvolution, probably the result of false CLEANing of sidelobe peaks in the dirty beam pattern. One can estimate the CLEAN bias by injecting artificial sources into the visibility data and compare the recovered flux densities after imaging \& deconvolution with the injected flux densities. We have not attempted this approach, but instead taken precautions to minimize the CLEAN bias effect. In our case, the dirty beam is well-behaved due to a relatively uniform UV-coverage from two extended observing runs, in combination with multi-frequency synthesis and a robust weighting parameter of 0.5 (slightly towards natural weighting). CLEAN bias is further suppressed through the use of tight CLEAN boxes in the imaging \& deconvolution process.

For an estimate of the contamination of the catalog with fake detections, we compare the GMRT $153 \mathrm{MHz}$ image and extracted source catalog against the results from the deep WSRT $1.4 \mathrm{GHz}$ survey of the Boötes field by de Vries et al. (2002). The $153 \mathrm{MHz}$ and $1.4 \mathrm{GHz}$ observations are well matched in terms of survey area and resolution ( $\sim 7$ square degrees and $13^{\prime \prime} \times 21^{\prime \prime}$ for WSRT, respectively). The typical noise over the WSRT survey area is $28 \mu \mathrm{Jy}_{\text {beam }^{-1}}$. For a spectral index of -0.8 , the $1.4 \mathrm{GHz}$ observations are $\sim 10$ times more sensitive. We restrict our comparison to a 1.4 degree radius circular area to avoid the noisy edge of the deep $1.4 \mathrm{GHz}$ survey. For all of the 399 sources detected at $153 \mathrm{MHz}$ we find a counterpart in the $1.4 \mathrm{GHz}$ map (383 sources were automatically matched within a $25^{\prime \prime}$ search radius, while the remaining fraction of sources with complex morphology were confirmed manually). We could not match the full GMRT area, but considering that our source extraction is based on local noise and that the false detections only appeared to occur near a few bright sources, we estimate that the contamination of our complete catalog over the full survey area is $<1$ percent.

\subsection{Astrometric and flux uncertainty}

For an estimate of the astrometric uncertainty, we compare the source positions in the GMRT $153 \mathrm{MHz}$ catalog against catalog source positions from the deep WSRT $1.4 \mathrm{GHz}$ map of de Vries et al. (2002). For our position comparison, we only use sources whose flux profile is accurately described by a single Gaussian, and whose peak flux $S_{\mathrm{p}}$ is at least $10 \sigma_{\mathrm{L}}$. This 
bypasses most of the position errors that arise from low signalto-noise $\left(\mathrm{S} / \mathrm{N}\right.$, or $\left.S_{\mathrm{p}} / \sigma_{\mathrm{L}}\right)$, different grouping of Gaussians and spectral variations across sources. Using a search radius of $10^{\prime \prime}$, we cross-match 126 sources in both catalogs. From this, we measure a small mean position offset in RA and Dec of $(\Delta \alpha, \Delta \delta)=\left(0.11^{\prime \prime}, 0.09^{\prime \prime}\right)$. We correct the catalog positions for this small offset. The estimated rms scatter around this offset is $\sigma_{\alpha, \delta}=1.32^{\prime \prime}$. The $\mathrm{S} / \mathrm{N}$-independent part of the positional uncertainty of the $1.4 \mathrm{GHz}$ sources is $0.44^{\prime \prime}$ (de Vries et al. 2002), therefore we derive an absolute astrometric uncertainty for the $153 \mathrm{MHz}$ sources of $1.24^{\prime \prime}$. We quadratically add this to the calculated (S/N-dependent) position accuracies in the $153 \mathrm{MHz}$ source catalog (see Sect. 3.1).

The uncertainty of the flux scale transferred from the calibrator 3C 286 to the target Boötes field is influenced by several factors: (i) the quality of the calibrator observational data, (ii) the accuracy of calibrator source model, and (iii) the difference in observing conditions between the calibrator and target field. Because of the relatively large uncertainty in the flux scale at low frequencies, we discuss in some detail the issues that influence these factors.

The quality of the calibrator data is most noticeably affected by RFI and by ionospheric phase rotations. The repeated observation of 3C 286 every $\sim 45$ min during the observing enabled us to monitor these effects over time. The mild fluctuations in the initial (short interval) calibration gain phases at the start of the data reduction showed that the ionosphere was very calm during both observing nights, therefore we exclude the possibility of diffraction or focussing effects (e.g., Jacobson \& Erickson 1992). Apparent flux loss due to ionospheric phase rotations was prevented by applying the (short interval) gain phase corrections before bandpass- and amplitude calibration (see Sect. 2.2).

RFI was continuously present during both observing sessions. This mainly consisted of persistent RFI over the full band, most noticeably on the shortest (central square and neighbouring arm antenna) baselines, and of more sporadic events on longer baselines during one or more time stamps and/or narrow frequency ranges. The sporadic events were relatively easy to recognize and excise, but for the persistent RFI this is much more difficult due to a lack of contrast between healthy and affected data on a single baseline. Some of the shortest, most affected baselines were removed completely. On longer baselines, persistent RFI from quasi-stationary sources can average out due to fringe tracking (Athreya 2009), but does add noise. Large magnitude RFI amplitude errors in the visibilities may result in a suppression of the gain amplitude corrections. Because these effects are hard to quantify, we adopt an ad-hoc 2 percent amplitude error due to RFI.

Because 3C 286 is unresolved $\left(\$ 2.5^{\prime \prime}\right)$ within a $20^{\prime \prime}$ to $25^{\prime \prime}$ beam, we used a point source for the calibrator model with a flux density of 31.01 Jy (Sect. 2.2). The utilized Perley-Taylor flux density at $153 \mathrm{MHz}$ is an extrapolatation of VLA flux density measurements at $330 \mathrm{MHz}$ and higher, using a fourth order polynomial in log-log space. In Table 4 a comparison is presented between flux density measurements of 3C 286 in various sky surveys at low frequencies and the predicted flux densities from the Perley-Taylor model. Although there is a large variation in the flux differences at the different frequencies, there appears to be a overestimation by the Perley-Taylor model below $200 \mathrm{MHz}$ due to a spectral turnover of 3C 286 below $~ 300 \mathrm{MHz}$. For this reason, we re-fitted the polynomial to the original data points plus the additional $74 \mathrm{MHz}$ VLSS measurement, assuming

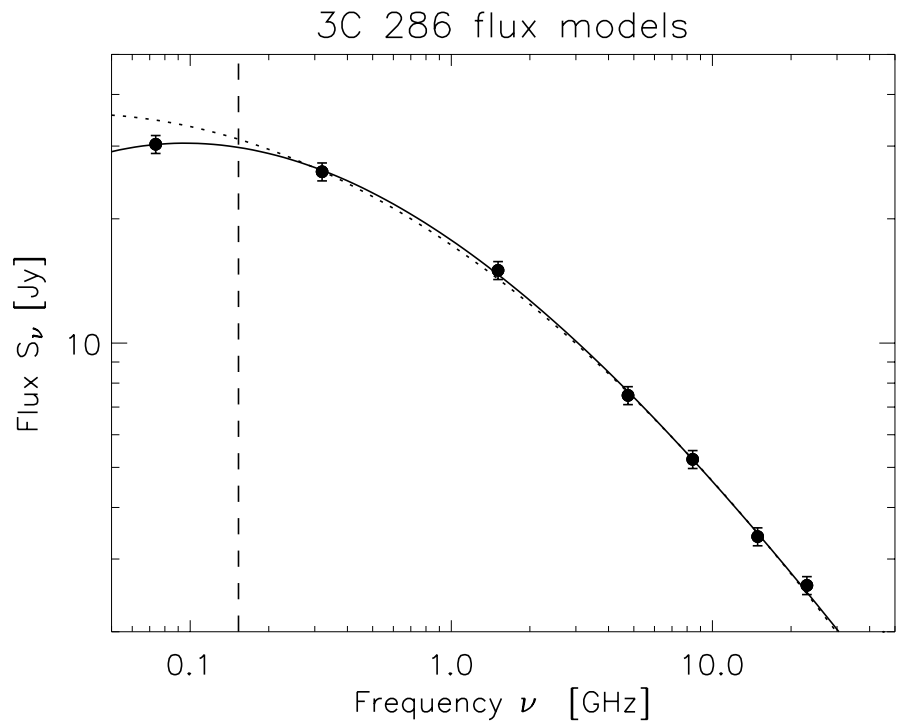

Fig. 3. Model of the radio spectrum of 3C 286. The measurements (dots) above $153 \mathrm{MHz}$ (dashed line) were used to fit the original Perley-Taylor 1999.2 model (dotted line), which appears to overestimate the flux density at $153 \mathrm{MHz}$. By adding one measurement at $74 \mathrm{MHz}$, we fit an alternative model (solid line) that may give a better prediction at $153 \mathrm{MHz}$.

5 percent errors for all data points (Fig. 3). Our new model is given by:

$$
\begin{aligned}
\log _{10}\left(S_{v}\right)= & 1.24922-0.434710 \log _{10}(v) \\
& -0.174786\left(\log _{10}(v)\right)^{2}+0.0251542\left(\log _{10}(v)\right)^{3},
\end{aligned}
$$

with $v$ the frequency in $\mathrm{GHz}$ and $S_{v}$ the flux density in Jy. This parametrization results in a slightly better fit to the flux densities from the various surveys (Table 4), although the large scatter remains. For the center of the GMRT band at $153.1 \mathrm{MHz}$, the new model predicts a flux density of $29.77 \mathrm{Jy}$. We have adopted this new model by scaling the image by the ratio $29.77 / 31.01$ before primary beam correction (see start of Sect. 3). The accuracy of this flux scale is closely related to the accuracy of the $74 \mathrm{MHz}$ data point, for which a 10 percent flux error is given. Assuming the error in the $330 \mathrm{MHz}$ is much smaller, and $153 \mathrm{MHz}$ is roughly half way from 74 to $330 \mathrm{MHz}$ in logarithmic frequency, we set an upper limit of 5 percent error on the adopted flux scale of $3 \mathrm{C} 286$ at $153 \mathrm{MHz}$.

The presence of other sources in the 3.1 degree FoV around 3C 286 further complicates the flux calibration. For example, the total apparent flux density in the Boötes field that was extracted through CLEAN deconvolution is $\sim 46 \mathrm{Jy}$, which may be typical lower limit for any blind field. If this flux is distributed over many sources that are individually much fainter than the calibrator, then the net effect of this additional flux is only noticeable on a small subset of the shortest baselines, while calibration utilizes all baselines. Inspection of the 3C 286 field at $74 \mathrm{MHz}$ (VLSS; Cohen et al. 2007) and $325 \mathrm{MHz}$ (WENSS; Rengelink et al. 1997) does identify two relatively bright sources within 0.7 degrees of 3C 286 with estimated apparent GMRT $153 \mathrm{MHz}$ flux densities of 5.3 and $2.7 \mathrm{Jy}$, respectively. These sources will cause a modulation of the visibility amplitudes across the UVplane. We performed a simple simulation, in which we replaced the measured 3C 286 visibilities with noise-less model visibilities of three point sources, being 3C 286 and the two nearby sources, and calibrated these visibilities against a single point source model of 3C 286, using the same settings as in the original data reduction. We found that the combined gain amplitude 
Table 4. Flux measurements of 3C 286 from different radio survey catalogs at low frequencies.

\begin{tabular}{lcccc}
\hline \hline Survey & $\begin{array}{c}\text { Frequency } \\
{[\mathrm{MHz}]}\end{array}$ & $\begin{array}{c}\text { Catalog flux } \\
{[\mathrm{Jy}]}\end{array}$ & $\begin{array}{c}\mathrm{PT}^{a} \text { model flux } \\
{[\mathrm{Jy}]}\end{array}$ & $\begin{array}{c}\text { New model flux } \\
{[\mathrm{Jy}]}\end{array}$ \\
\hline $\mathrm{VLSS}^{b}$ & 74 & $30.26 \pm 3.08$ & 34.67 & 30.26 \\
CC $^{c}$ & 151 & 26.31 & 31.25 & 29.81 \\
$7 \mathrm{C}^{d}$ & 151 & 26.53 & 31.25 & 29.81 \\
$3 \mathrm{C}^{e}$ & 159 & $30.0 \pm 7.0$ & 30.94 & 29.65 \\
$4 \mathrm{C}^{f}$ & 178 & 24.0 & 30.22 & 29.26 \\
WENSS $^{g}$ & 325 & $27.12 \pm 1.63$ & 25.96 & 26.11 \\
\hline
\end{tabular}

References. ${ }^{(a)}$ Perley-Taylor. ${ }^{(b)}$ Cohen et al. (2007). ${ }^{(c)}$ Hales et al. (1988). ${ }^{(d)}$ Hales et al. (2007) and references therein. Note that we use the peak flux rather than the integrated flux density, as 3C 286 is unresolved on the 7C resolution. ${ }^{(e)}$ Edge et al. (1959); Bennett (1962). ${ }^{(f)}$ Pilkington \& Scott (1965); Gower et al. (1967). ${ }^{(g)}$ Rengelink et al. (1997). Note that we use the peak flux rather than the integrated flux, as 3C 286 is unresolved on the WENSS resolution.

for all antennas and all time intervals was $1.000 \pm 0.004$. For individual $10 \mathrm{~min}$ time blocks, the largest deviation from one was 1.00 percent, which indicates the magnitude of the possible error when using a single 10 min calibrator observation on $3 \mathrm{C}$ 286. The small deviations per time block are transferred to the target field data, but these are suppressed by amplitude selfcalibration against the target field source model. We set an upper limit of 1 percent due to the presence of other sources in the FoV of the calibrator.

While transferring the flux scale from calibrator to target field, the derived gain amplitudes need to be corrected for differences in sky temperature due to galactic diffuse radio emission (e.g., Tasse et al. 2007), which is detected by individual array antennas but not by the interferometer. The GMRT does not implement a sky temperature measurement, therefore we need to rely on an external source of information. From the Haslam et al. (1982) all-sky map, we find that the mean off-source sky temperatures at $408 \mathrm{MHz}$ as measured in the 3C 286 and Boötes field are both approximately $20 \pm 1$ degree. Applying the formulae given by Tasse et al. (2007) for the GMRT at $153 \mathrm{MHz}^{6}$, we estimate a gain inaccuracy of $\sim 2$ percent at most.

Another effect that may influence the gain amplitude transfer between calibrator and target field is an elevation-dependent gain error. This is a combination of effects such as structural deformation of the antenna, atmospheric refraction and changes in system temperature from ground radiation. According to Chandra et al. (2004), the effect on amplitude is rather small, if not negligible, for GMRT frequencies of $610 \mathrm{MHz}$ and below. Furthermore, the relatively short angular distance of 13.4 degrees between 3C 286 and the target field center causes the differential elevation error to be limited. Elevation dependent phase errors are not relevant, because we don't rely on the calibrator to restore the astrometry. For our observation, we assume that elevation-dependent effects can be ignored.

To incorporate the effects discussed above plus some margin, we quadratically add a relative flux error of 10 percent to the flux density measurement errors in the source catalog.

\section{Analysis}

The 598 radio sources in our $153 \mathrm{MHz}$ catalog form a statistically significant set, ranging in flux density from $5.1 \mathrm{mJy}$ to 3.9 Jy. Because of the large survey area, cosmic variance is expected to be small. Radio images at $153 \mathrm{MHz}$ of a selection of extended sources are presented in Appendix A. In this section,

\footnotetext{
${ }^{6}$ We adopted the GMRT system parameters from http://wwW . gmrt.ncra.tifr.res.in/gmrt_hpage/Users/doc/manual/ UsersManual/node13.html
}

Table 5. Euclidean-normalized differential source counts (including Poissonian error estimates) for the full $153 \mathrm{MHz}$ catalog of 598 sources, distributed over 14 logarithmic flux bins ranging from $4.75 \mathrm{mJy}$ to $3 \mathrm{Jy}$.

\begin{tabular}{ccc}
\hline $\begin{array}{c}\text { Flux bin center } \\
{[\mathrm{Jy}]}\end{array}$ & Raw counts & $\begin{array}{c}\text { Normalized counts } \\
{\left[\mathrm{Jy}^{3 / 2} \mathrm{sr}^{-1}\right]}\end{array}$ \\
\hline 0.0060 & 30 & $58.70 \pm 14.12$ \\
0.0095 & 89 & $105.05 \pm 14.92$ \\
0.0150 & 102 & $133.90 \pm 14.18$ \\
0.0238 & 87 & $199.81 \pm 21.45$ \\
0.0378 & 76 & $347.09 \pm 39.81$ \\
0.0599 & 60 & $546.74 \pm 70.58$ \\
0.0945 & 57 & $1036.35 \pm 137.27$ \\
0.1504 & 36 & $1305.98 \pm 217.66$ \\
0.2383 & 19 & $1375.27 \pm 315.51$ \\
0.3777 & 16 & $2310.75 \pm 577.69$ \\
0.5986 & 11 & $3169.75 \pm 955.72$ \\
0.9487 & 6 & $3449.72 \pm 1408.34$ \\
1.5036 & 7 & $8030.29 \pm 3035.16$ \\
2.3830 & 1 & $2288.93 \pm 2288.93$ \\
\hline
\end{tabular}

we discuss two characteristics of the survey: number counts and spectral indices. Further analyses will be done in a subsequent article. When properly corrected for incompleteness, the number counts are an objective measure of the $153 \mathrm{MHz}$ source population, that can be compared against models and other surveys. For individual sources, the spectral index can help to classify sources, and identify rare sources such as HzRGs and cluster halos \& relics.

\subsection{Differential source counts}

We derived the Euclidean-normalized differential source counts from the catalog. Because the source extraction criteria vary over the survey area, we used Fig. 2 to correct for the missed fraction per flux bin. This mainly affects the lowest two bins. Furthermore, the combined effect of random peaks in the background noise and a peak detection criteria causes a selection bias for positively enhanced weak sources (Eddington bias). In general, noise can scatter sources into other flux bins, most noticeably near the detection limit. Our attempts to correct for this effect through Monte-Carlo simulations were numerically unstable due to the low number counts in the lowest flux bin. The effect on the higher bins was minimal, therefore we omit the Eddington bias corrections. The Euclidean normalized differential source counts are tabulated in Table 5. Figure 4 shows our source counts in comparison with the (little) other observational data that is available for this frequency at comparable sensitivity, as well as source counts derived from two models. 


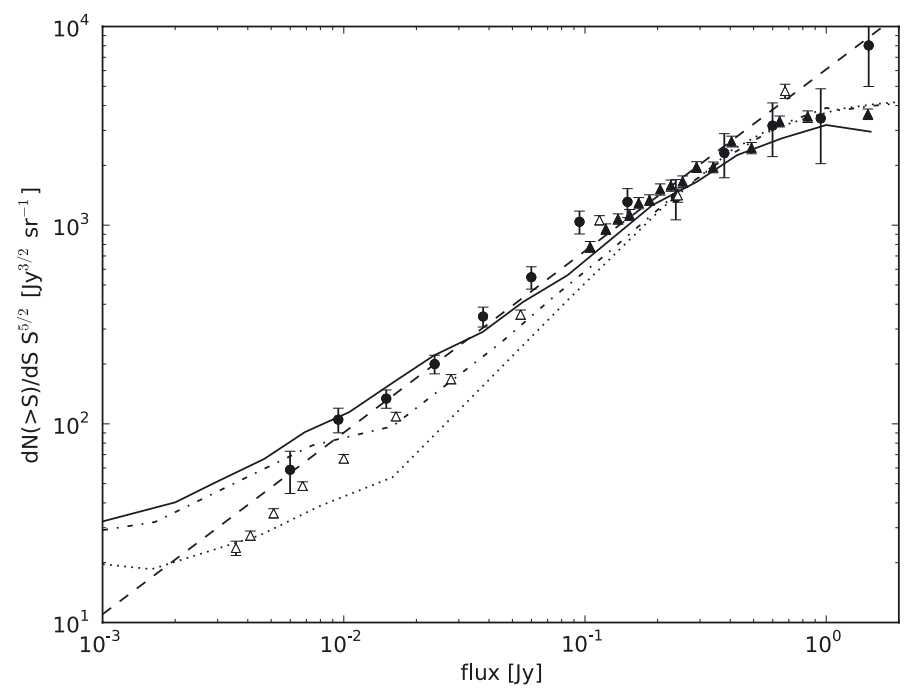

Fig. 4. Euclidean-normalized differential source counts for the full $153 \mathrm{MHz}$ catalog of 598 sources, distributed over 14 logarithmic flux bins ranging from $4.75 \mathrm{mJy}$ to $3 \mathrm{Jy}$ (black dots; we have omitted the highest flux bin, containing just one count). Also plotted are $151 \mathrm{MHz}$ source counts by McGilchrist et al. (1990), part of the 7C catalog (black triangles), $153 \mathrm{MHz}$ source counts by Ishwara-Chandra et al. (2010, white triangles), model $151 \mathrm{MHz}$ source counts by Jackson (2005) for a $\Lambda$ CDM cosmology (dotted line) and an $\Omega_{\mathrm{m}}=1$ cosmology (dashdotted line), a $151 \mathrm{MHz}$ source count model by Wilman et al. (2008, solid line), and the power-law slope of 0.91 (dashed line) that fits our data points best.

At the high flux end $(0.1-1 \mathrm{Jy})$, there is a good agreement between our source counts and those derived from part of the 7C survey at $151 \mathrm{MHz}$ (McGilchrist et al. 1990). Ishwara-Chandra et al. (2010) present source counts of 765 sources in a singlefield, deep GMRT $153 \mathrm{MHz}$ observation similar to ours, but with over twice the bandwidth $(16 \mathrm{MHz})$. Their source counts go down to $\sim 2.5 \mathrm{mJy}$. While their source counts are roughly equal to ours at the high flux end, they are increasingly lower towards lower flux levels. Their source counts are well approximated by a single power-law slope of 1.01 , which is steeper than the value of 0.91 from our data over the range $40-400 \mathrm{mJy}$. This suggests a non-linear flux scale difference. We can offer little explanation on this apparent discrepancy. Within 2-3 times the $(1 \sigma)$ error bars, the two source count results may still be considered consistent.

George \& Stevens (2008) have determined source counts from a shallower GMRT $153 \mathrm{MHz}$ field (central rms $3.1 \mathrm{mJy}$ beam $^{-1}$ ) centered around $\epsilon$ Eridanus. From binning 113 source fluxes over a range of $20 \mathrm{mJy}-2$ Jy they derive a single power-law slope of 0.72 (not plotted). The most likely explanation for this much flatter slope is the large uncertainties in their counts due to low-number statistics.

Jackson (2005) constructed a $151 \mathrm{MHz}$ source count model, based on an extrapolation of source counts $>0.2$ Jy from the 3CRR and 6C catalogs (Laing et al. 1983; Hales et al. 1988). Two cosmological scenarios are considered, namely an $\Omega_{\mathrm{m}}=1$ cosmology and a $\Lambda$ CDM cosmology, the latter being today's generally accepted cosmology (e.g., Komatsu et al. 2011). In this model, FRII radio sources dominate the counts above $\sim 50$ and $\sim 20 \mathrm{mJy}$, for the two cosmologies respectively. Below, FRI sources are the most dominant population down to below our detection threshold, which causes a flattening of the counts below $\sim 20 \mathrm{mJy}$ in both cosmologies.
Figure 4 shows that the source count models for both scenarios roughly match our observed source counts near $500 \mathrm{mJy}$, but increasingly underestimate the counts towards lower flux densities. Between 20 and $200 \mathrm{mJy}$, the approximately constant model slope for both scenarios is 0.98 and 1.19 , respectively, steeper than the value of 0.91 derived from our data. The observed source counts shows no clear evidence of flattening towards lower flux levels.

Wilman et al. (2008) have generated model source counts at $151 \mathrm{MHz}$ from a semi-empirical $(\Lambda \mathrm{CDM})$ cosmological simulation, using (extrapolated) radio luminosity functions for several different populations of sources. In this model, FRII radio sources dominate the counts above $\sim 200 \mathrm{mJy}$, while below FRI sources dominate down to beyond our detection threshold. Figure 4 shows an approximate agreement between the model and our observations, although the average power-law slope between 10 and $200 \mathrm{mJy}$ of 0.83 is flatter than the value of 0.91 derived from our data.

For the models that are discussed here we find that all predicted source counts deviate from the observed source counts. The model source count slopes appear to be influenced mostly by the assumed contribution of FRI sources. On the other hand, the observational results from Ishwara-Chandra et al. (2010) also deviate from our result. More source counts from similar deep surveys at this frequency are needed to put stronger constraints on the source count models. Preliminary source counts from a deep GMRT $153 \mathrm{MHz}$ survey three times the area of our current survey (>1000 sources; Intema et al., in prep.) match up closely with our current results. And future source counts from the ongoing GMRT $153 \mathrm{MHz}$ sky survey (TGSS) should provide a more robust reference to compare the source count models and our results against.

\subsection{Spectral Indices}

Because of the good match in resolution between the GMRT $153 \mathrm{MHz}$ image and the deep WSRT $1.4 \mathrm{GHz}$ image from de Vries et al. (2002), we can accurately determine spectral indices over a decade in frequency. The survey depths at $153 \mathrm{MHz}$ and $1.4 \mathrm{GHz}$ are equal for sources with a spectral index of -1.6 . Due to the high detection rate of $1.4 \mathrm{GHz}$ sources at $153 \mathrm{MHz}$ positions (Sect. 3.2), we do an automated search for $1.4 \mathrm{GHz}$ counterparts within $25^{\prime \prime}$ of the $153 \mathrm{MHz}$ sources and ignore all sources for which we don't find counterparts. The spectral indices of 417 matched sources are plotted in Fig. 5. We find a median spectral index of -0.76 , which is similar to the median values of -0.79 (Cohen et al. 2004), -0.85 (Ishwara-Chandra \& Marathe 2007), -0.82 (Sirothia et al. 2009) and -0.78 (IshwaraChandra et al. 2010) found for similar high-resolution, lowfrequency surveys at 74 and $153 \mathrm{MHz}$. The small differences are most likely caused by differences in the completeness limits of the catalogs, as the median spectral index varies with flux density (see below).

Figure 5 also plots the median spectral index in 6 logarithmic flux bins, which clearly highlights the flattening trend of the mean spectral index towards lower flux densities. The unique combination of our deep $153 \mathrm{MHz}$ catalog and the very deep $1.4 \mathrm{GHz}$ catalog makes that the median spectral index is unbiased down to the lowest $153 \mathrm{MHz}$ flux densities. The median spectral index is $\sim-0.9$ for $\gtrsim 0.5 \mathrm{Jy}$ sources, and flattens to $\sim-0.7$ for $\lesssim 50$ mJy sources. A similar trend appears to be present in the spectral index distribution by Ishwara-Chandra et al. (2010) for sources between $153 \mathrm{MHz}$ and various frequencies $(610 \mathrm{MHz}$ and higher), but may be biased by catalog flux limits at the higher 
H. T. Intema et al.: Deep low-frequency radio observations of the Boötes field. I.

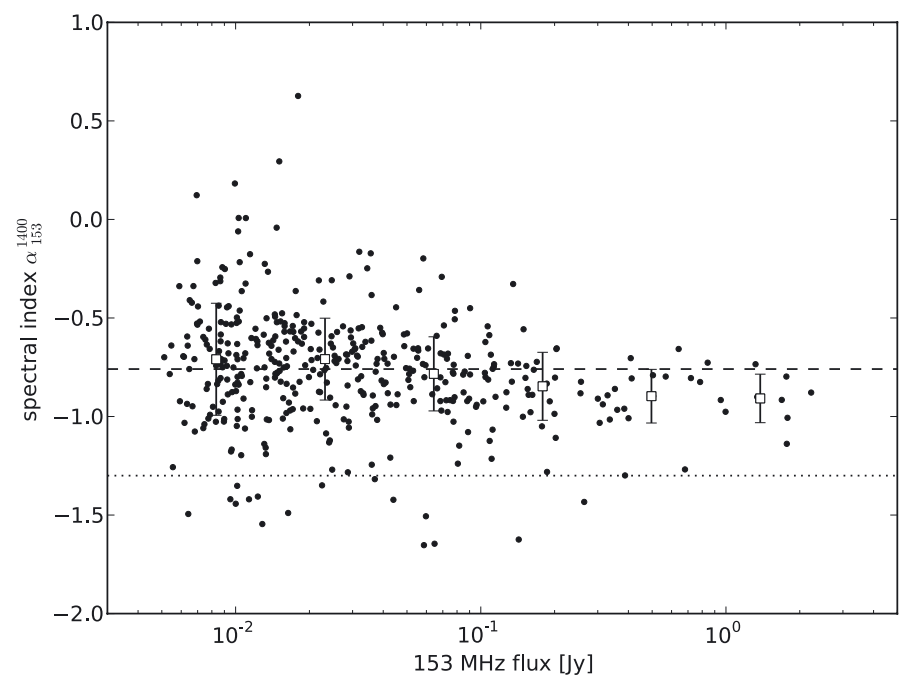

Fig. 5. Spectral index between $1.4 \mathrm{GHz}$ and $153 \mathrm{MHz}$ for 417 sources, plotted as a function of the $153 \mathrm{MHz}$ flux (black dots). The detection limit is almost fully determined by the $153 \mathrm{MHz}$ survey due to the sensitivity of the $1.4 \mathrm{GHz}$ observations. The median spectral index is -0.76 (dashed line). 16 sources have a spectral index below -1.3 (dotted line). The spectral indices are binned to emphasize the spectral flattening towards lower flux densities (white squares; see Table 6).

Table 6. Median spectral index between $1.4 \mathrm{GHz}$ and $153 \mathrm{MHz}$ for 417 sources in 6 logarithmic $(153 \mathrm{MHz})$ flux bins ranging from $5.0 \mathrm{mJy}$ to $2.3 \mathrm{Jy}$.

\begin{tabular}{ccc}
\hline $\begin{array}{c}\text { Flux bin center } \\
{[\mathrm{Jy}]}\end{array}$ & Counts & Spectral index $^{a}$ \\
\hline 0.0083 & 133 & $-0.710 \pm 0.284$ \\
0.0232 & 133 & $-0.709 \pm 0.208$ \\
0.0643 & 84 & $-0.784 \pm 0.188$ \\
0.1786 & 38 & $-0.847 \pm 0.173$ \\
0.4966 & 19 & $-0.897 \pm 0.136$ \\
1.3799 & 10 & $-0.908 \pm 0.123$ \\
\hline
\end{tabular}

Notes. ${ }^{(a)}$ Median \pm rms around median.

frequencies. A similar flattening trend is also seen at both lower and higher frequencies, e.g., between $74 \mathrm{MHz}$ and $1.4 \mathrm{GHz}$ by Cohen et al. (2004) and Tasse et al. (2006), between $1.4 \mathrm{GHz}$ and $325 \mathrm{MHz}$ by de Vries et al. (2002), between $325 \mathrm{MHz}$ and $1.4 \mathrm{GHz}$ by Zhang et al. (2003) and Owen et al. (2009), between $610 \mathrm{MHz}$ and $1.4 \mathrm{GHz}$ by Bondi et al. (2007), and between 1.4 and $5 \mathrm{GHz}$ by Prandoni et al. (2006).

Recently, Bornancini et al. (2010) found that, for a nearcomplete sample of radio galaxies, there is no evidence for spectral steepening or flattening due to redshifted curved radio spectra. In fact, most of their sources have straight power-law spectra from $74 \mathrm{MHz}$ to $4.8 \mathrm{GHz}$. This suggests that the observed spectral flattening towards lower flux densities results dominantly from a correlation between source luminosity and spectral in$\operatorname{dex}(P-\alpha$ correlation). This correlation is found to exist for FRII galaxies (e.g., Blundell et al. 1999). According to the models by Jackson (2005) and Wilman et al. (2008), FRII galaxies are the dominant $153 \mathrm{MHz}$ source population in our sample at higher flux levels ( $220-200 \mathrm{mJy}$ ), which can explain part of the flattening observation. For lower flux levels, the flattening trend continues, which suggests that either FRII galaxies are still a significant source population, or the FRI galaxies dominate and also follow a $P-\alpha$ correlation.
From Fig. 5 we highlight a small group of 16 USS sources that have a spectral indices lower than -1.3 . The lowest spectral index is -1.65 , which indicates that the sample selection limit is indeed dominated by the $153 \mathrm{MHz}$ survey depth. Table 7 lists these sources in decreasing flux order. Despite the relatively large uncertainty in $153 \mathrm{MHz}$ flux density for the faintest sources, the spectral index is still relatively well constrained due to the large frequency span.

The fraction of USS sources is 3.8 percent (16 out of 417 ), which is mostly dependent on the survey detection limit at $153 \mathrm{MHz}$. It is not straightforward to compare this fraction with the few other $153 \mathrm{MHz}$ surveys, as they use different high frequencies, with different detection limits at the high- and lowfrequency end. Using the same criteria, Sirothia et al. (2009) find a USS fraction of 3.7 percent (14 out of 374). Applying the same criteria to the source catalog by Ishwara-Chandra et al. (2010) yields a USS fraction of 3.0 percent (19 out of 639). These three fractions appear to have some consistency.

The angular distribution of the USS sources is quite peculiar. There are 6 sources that form 3 pairs within $6^{\prime}$ of each other. These pairs, together with the remaining 10 single sources, appear to be randomly distributed across the FoV. Visual inspection of the pairs in the images at $153 \mathrm{MHz}$ and $1.4 \mathrm{GHz}$ did not reveal any obvious artifacts in the background near these sources, which makes it less likely that these sources are fake detections. There is no visual evidence in the radio maps that the pair components are physically connected. Through Monte-Carlo simulations we determined that, out of 16 sources with random positions over a 1.5 degree radius field (which is approximately the $1.4 \mathrm{GHz}$ field radius), the chance of finding 3 pairs within $6^{\prime}$ is $1.4 \pm 0.4$ percent. Therefore, it is unlikely that these pairs appeared by chance. Further investigation is needed to establish the true nature of these pairs.

Croft et al. (2008) examine 4 candidate HzRGs (which they labelled A, B, C and E) based on their steep $(\leq-0.87)$ spectral index between $1.4 \mathrm{GHz}$ and $325 \mathrm{MHz}$. As their candidate sources are also present in the $153 \mathrm{MHz}$ catalog, we complement their data with our new spectral index measurements in Table 8. Both sources A and E appear to have fairly straight power-law spectra down to $153 \mathrm{MHz}$, while sources $\mathrm{B}$ and $\mathrm{C}$ appear to undergo considerable spectral flattening. Based on our selection criteria for USS sources, only source A would be considered a candidate HzRG.

\subsection{Identification fraction of radio sources versus spectral index}

We investigate the NIR $K$-band identification fraction of radio sources as function of spectral index (e.g., Wieringa 1991). This links together two known correlations, namely the correlation between spectral index and redshift (Tielens et al. 1979; Blumenthal \& Miley 1979) and the correlation between $K$-band magnitude and redshift $(K-z$ correlation; e.g., Willott et al. 2003; Rocca-Volmerange et al. 2004). We remark that the correlation between spectral index and redshift is not a tight correlation; for a given spectral index there is a range of possible redshifts for a radio source (e.g., Miley \& De Breuck 2008). But on average, steep spectrum sources are located at higher redshifts, and are consequently (on average) more difficult to detect in optical and NIR bands. Using $K$-band has an advantage over (also available) shorter wavelength bands as this band suffers the least from extinction. 
Table 7. $153 \mathrm{MHz}$ catalog selection of 16 USS sources with a spectral index $\alpha_{153}^{1400}$ below -1.3 .

\begin{tabular}{lccccc}
\hline \hline $\mathrm{ID}^{a}$ & $\mathrm{RA}^{b}$ & $\operatorname{Dec}^{b}$ & $\begin{array}{c}153 \mathrm{MHz} \text { flux } \\
{[\mathrm{mJy}]}\end{array}$ & $\begin{array}{c}1.4 \mathrm{GHz} \text { flux } \\
{[\mathrm{mJy}]}\end{array}$ & $\alpha_{153}^{1400}$ \\
\hline $\mathrm{J} 142656+352230$ & $14^{\mathrm{h}} 26^{\mathrm{m}} 56.46^{\mathrm{s}}$ & $35^{\circ} 22^{\prime} 30.8^{\prime \prime}$ & $264.2 \pm 26.6$ & $11.31 \pm 0.46$ & $-1.43 \pm 0.05$ \\
$\mathrm{~J} 143506+350059$ & $14^{\mathrm{h}} 35^{\mathrm{m}} 06.89^{\mathrm{s}}$ & $35^{\circ} 00^{\prime} 58.2^{\prime \prime}$ & $142.8 \pm 14.5$ & $4.02 \pm 0.16$ & $-1.62 \pm 0.05$ \\
$\mathrm{~J} 143118+351549$ & $14^{\mathrm{h}} 31^{\mathrm{m}} 18.29^{\mathrm{s}}$ & $35^{\circ} 15^{\prime} 49.5^{\prime \prime}$ & $64.8 \pm 6.9$ & $1.74 \pm 0.08$ & $-1.65 \pm 0.05$ \\
$\mathrm{~J} 143500+342531$ & $14^{\mathrm{h}} 35^{\mathrm{m}} 00.98^{\mathrm{s}}$ & $34^{\circ} 25^{\prime} 30.2^{\prime \prime}$ & $59.7 \pm 6.2$ & $2.18 \pm 0.09$ & $-1.51 \pm 0.05$ \\
$\mathrm{~J} 143520+345950$ & $14^{\mathrm{h}} 35^{\mathrm{m}} 20.51^{\mathrm{s}}$ & $34^{\circ} 59^{\prime} 49.1^{\prime \prime}$ & $58.6 \pm 6.2$ & $1.55 \pm 0.07$ & $-1.65 \pm 0.05$ \\
$\mathrm{~J} 143815+344428$ & $14^{\mathrm{h}} 38^{\mathrm{m}} 15.28^{\mathrm{s}}$ & $34^{\circ} 44^{\prime} 29.8^{\prime \prime}$ & $44.0 \pm 4.8$ & $1.93 \pm 0.09$ & $-1.42 \pm 0.05$ \\
$\mathrm{~J} 143331+341012$ & $14^{\mathrm{h}} 33^{\mathrm{m}} 31.84^{\mathrm{s}}$ & $34^{\circ} 10^{\prime} 12.9^{\prime \prime}$ & $37.0 \pm 4.1$ & $2.04 \pm 0.09$ & $-1.32 \pm 0.05$ \\
$\mathrm{~J} 142631+341557$ & $14^{\mathrm{h}} 26^{\mathrm{m}} 31.69^{\mathrm{s}}$ & $34^{\circ} 16^{\prime} 00.9^{\prime \prime}$ & $22.5 \pm 3.6$ & $1.16 \pm 0.07$ & $-1.35 \pm 0.08$ \\
$\mathrm{~J} 142954+343516$ & $14^{\mathrm{h}} 29^{\mathrm{m}} 53.90^{\mathrm{s}}$ & $34^{\circ} 35^{\prime} 18.8^{\prime \prime}$ & $16.4 \pm 2.4$ & $0.62 \pm 0.04$ & $-1.49 \pm 0.07$ \\
$\mathrm{~J} 142724+334714$ & $14^{\mathrm{h}} 27^{\mathrm{m}} 24.77^{\mathrm{s}}$ & $33^{\circ} 47^{\prime} 18.5^{\prime \prime}$ & $12.8 \pm 2.8$ & $0.43 \pm 0.04$ & $-1.55 \pm 0.11$ \\
$\mathrm{~J} 143538+335347$ & $14^{\mathrm{h}} 35^{\mathrm{m}} 38.76^{\mathrm{s}}$ & $33^{\circ} 53^{\prime} 44.2^{\prime \prime}$ & $12.3 \pm 2.2$ & $0.56 \pm 0.05$ & $-1.41 \pm 0.09$ \\
$\mathrm{~J} 143310+333131$ & $14^{\mathrm{h}} 33^{\mathrm{m}} 10.42^{\mathrm{s}}$ & $33^{\circ} 31^{\prime} 27.7^{\prime \prime}$ & $11.3 \pm 2.2$ & $0.50 \pm 0.04$ & $-1.42 \pm 0.10$ \\
$\mathrm{~J} 143230+343449$ & $14^{\mathrm{h}} 32^{\mathrm{m}} 30.47^{\mathrm{s}}$ & $34^{\circ} 34^{\prime} 49.5^{\prime \prime}$ & $10.1 \pm 1.8$ & $0.52 \pm 0.04$ & $-1.35 \pm 0.09$ \\
$\mathrm{~J} 142719+352326$ & $14^{\mathrm{h}} 27^{\mathrm{m}} 19.32^{\mathrm{s}}$ & $35^{\circ} 23^{\prime} 29.2^{\prime \prime}$ & $10.0 \pm 3.4$ & $0.42 \pm 0.06$ & $-1.44 \pm 0.17$ \\
$\mathrm{~J} 143700+335920$ & $14^{\mathrm{h}} 37^{\mathrm{m}} 00.74^{\mathrm{s}}$ & $33^{\circ} 59^{\prime} 20.2^{\prime \prime}$ & $9.5 \pm 2.5$ & $0.42 \pm 0.04$ & $-1.42 \pm 0.13$ \\
$\mathrm{~J} 143249+343915$ & $14^{\mathrm{h}} 32^{\mathrm{m}} 49.12^{\mathrm{s}}$ & $34^{\circ} 39^{\prime} 14.0^{\prime \prime}$ & $6.4 \pm 1.8$ & $0.24 \pm 0.04$ & $-1.49 \pm 0.15$ \\
\hline
\end{tabular}

Notes. ${ }^{(a)}$ From de Vries et al. (2002). ${ }^{(b)}$ Measured at $153 \mathrm{MHz}$.

Table 8. Spectral indices between $1.4 \mathrm{GHz}$ and $153 \mathrm{MHz}$ for four candidate HzRGs by Croft et al. (2008).

\begin{tabular}{lccccc}
\hline \hline ID & RA $^{a}$ & $\operatorname{Dec}^{a}$ & $\alpha_{325}^{1400 b}$ & $\alpha_{153}^{1400 c}$ & $z_{\text {phot }}{ }^{d}$ \\
\hline A & $14^{\mathrm{h}} 26^{\mathrm{m}} 31.75^{\mathrm{s}}$ & $+34^{\circ} 15^{\prime} 57.5^{\prime \prime}$ & -1.48 & $-1.35 \pm 0.08$ & 4.97 \\
B & $14^{\mathrm{h}} 26^{\mathrm{m}} 47.87^{\mathrm{s}}$ & $+34^{\circ} 58^{\prime} 51.0^{\prime \prime}$ & -0.89 & $-0.56 \pm 0.06$ & 3.76 \\
$\mathrm{C}$ & $14^{\mathrm{h}} 27^{\mathrm{m}} 41.84^{\mathrm{s}}$ & $+34^{\circ} 23^{\prime} 24.7^{\prime \prime}$ & -0.98 & $-0.44 \pm 0.13$ & 1.21 \\
$\mathrm{E}$ & $14^{\mathrm{h}} 32^{\mathrm{m}} 58.44^{\mathrm{s}}$ & $+34^{\circ} 20^{\prime} 55.4^{\prime \prime}$ & -0.87 & $-0.95 \pm 0.05$ & 4.65 \\
\hline
\end{tabular}

Notes. ${ }^{(a)}$ As measured at $1.4 \mathrm{GHz}$; de Vries et al. (2002). ${ }^{(b)}$ The spectral index between $325 \mathrm{MHz}$ and $1.4 \mathrm{GHz}$ as measured by Croft et al. (2008). ${ }^{(c)}$ The spectral index between $153 \mathrm{MHz}$ and $1.4 \mathrm{GHz}$ as measured in this work. ${ }^{(d)}$ Photometric redshift, as measured by Croft et al. (2008).

We have identified possible optical counterparts of the radio sources using the FLAMEX $K_{\mathrm{S}}$-band catalogue (Elston et al. 2006). This survey covers 7.1 square degrees within the Boötes field. For the NIR identification we use the likelihood ratio technique described by Sutherland \& Saunders (1992). This allows us to obtain an association probability for each NIR counterpart, taking into account the NIR magnitudes of the possible counterparts. Before the cross identification, we removed all radio sources located outside the coverage area of the $K_{\mathrm{S}}$-band images, or located within 20 pixels of the edge or other blanked pixels within individual $K_{\mathrm{S}}$-band frames.

Following Sutherland \& Saunders (1992) and Tasse et al. (2008), the probability that a NIR counterpart with magnitude $m$ is the true NIR counterpart of the radio source is given by the likelihood ratio

$\operatorname{LR}(r, m)=\frac{\theta(<m) \exp \left(-r^{2} / 2\right)}{2 \pi \sigma_{\alpha} \sigma_{\delta} \rho(<m)}$,

with $m$ the $K_{\mathrm{S}}$-band magnitude of the NIR candidate, $\theta(<m)$ the a priori probability that the radio source has a NIR counterpart with a magnitude brighter than $m$, and $\rho(<m)$ the surface number density of NIR sources with a magnitude smaller than $m$. $\sigma_{\delta}^{2}$ and $\sigma_{\alpha}^{2}$ are the quadratic sums of the uncertainties in the radio and NIR positions in right ascension and declination, respectively. For the radio source positions and uncertainties we have taken the values from the $1.4 \mathrm{GHz}$ WSRT catalogue, as the astrometric precision is better than the $153 \mathrm{MHz}$ GMRT data. For the FLAMEX survey we have adopted $0.3^{\prime \prime}$ for the position uncertainty (this is half the CCD pixel size, as source positions are truncated to pixel positions). The uncertaintynormalized angular distance is given by the parameter $r=$ $\sqrt{\left(\Delta_{\alpha} / \sigma_{\alpha}\right)^{2}+\left(\Delta_{\delta} / \sigma_{\delta}\right)^{2}}$, with $\Delta$ the positional difference in either $\alpha$ or $\delta$ between the possible NIR counterpart and radio source.

The probability $P(i)$ that candidate $i$ is the true NIR counterpart is

$$
P(i)=\frac{\operatorname{LR}_{i}(r, m)}{\sum_{j} \operatorname{LR}_{j}(r, m)+\left[1-\theta\left(m_{\text {lim }}\right)\right]},
$$

with $j$ running over all possible NIR counterparts. $\theta\left(m_{\lim }\right)$ is the fraction of radio sources having a NIR counterpart at the magnitude limit of the NIR survey. We found $\theta\left(m_{\text {lim }}\right)$ to be 0.62 , where $m_{\lim }=19.2$. The values for $\rho(<m)$ (surface number density of NIR sources) were estimated from the data itself using bins of $0.1 \mathrm{mag}$ across the full survey area. The a priori identification fraction $\theta(<m)$ was also estimated from the data itself, using the technique described by Ciliegi et al. (2003). This involves counting the surface number density of NIR sources around the radio sources as function of magnitude. This distribution is compared to a distribution of background objects, the overdensity of NIR sources around radio sources gives an estimate of $\theta(<m)$.

We selected 368 radio sources that are present within both the $1.4 \mathrm{GHz}$ and $153 \mathrm{MHz}$ catalogs (matched within 6") and have a simple morphology (fitted with a single Gaussian). We have computed the likelihood ratio of all NIR counterparts located within $20^{\prime \prime}$ from the radio position. We have defined a radio source to have a NIR counterpart if $\sum_{j} \operatorname{LR}_{j}(r, m)>0.75$. The results are shown in Fig. 6. The identification fraction is $\sim 70$ percent for $\alpha_{153}^{1400}>-0.7$, while for $\alpha_{153}^{1400}<-0.7$ the identification 


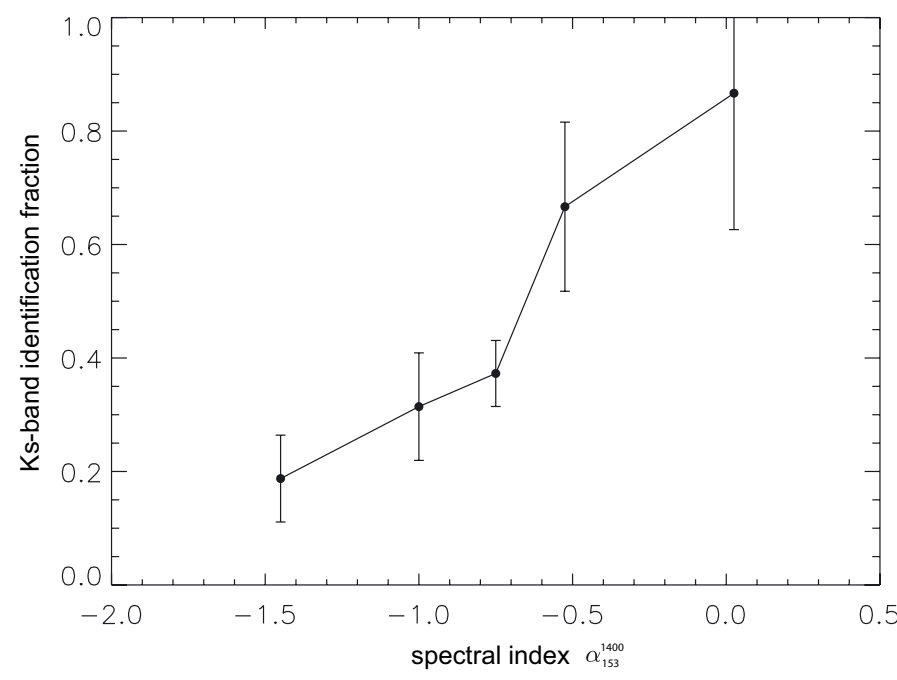

Fig. 6. Identification fraction of radio sources in the near-infrared $K_{\mathrm{S}}$ band as a function of spectral index between $1.4 \mathrm{GHz}$ and $153 \mathrm{MHz}$.

fraction drops to about 30 percent. This result reproduces the expected (and previously observed) correlation between spectral index and $K$-band identification fraction (e.g., Wieringa 1991).

\section{Conclusions and future plans}

We have presented the results from a deep $(\sim 1.0 \mathrm{mJy}$ central noise), high-resolution $\left(26^{\prime \prime} \times 22^{\prime \prime}\right)$ radio survey at $153 \mathrm{MHz}$, covering the full NOAO Boötes field and beyond. This 11.3 square degree survey is amongst the deepest surveys at this frequency to date. We produced a catalog of 598 sources detected at 5 times the local noise level, with source flux densities ranging from $3.9 \mathrm{Jy}$ down to $5.1 \mathrm{mJy}$. We estimate the catalog to be $\sim 70$ percent complete at the $5.1 \mathrm{mJy}$ flux limit, $\sim 92$ percent for $>10 \mathrm{mJy}$ sources and $>99$ percent for $>20 \mathrm{mJy}$ sources, and less than 1 percent contaminated by false detections. The onsource dynamic range (with noise measured near the source) is limited to $\sim 600$, while off-source (noise measured in central part of the image, away from bright sources) this rises to $>1000$. We expect that residual RFI and residual direction-dependent calibration errors prevents reaching the thermal noise level of $0.2-$ $0.3 \mathrm{mJy}$ beam $^{-1}$.

The $153 \mathrm{MHz}$ catalog presented in Sect. 3.1 allows for a detailed study of source populations in a relatively unexplored flux range. We have analyzed the source counts and spectral index distributions for our survey. From this analysis we draw the following conclusions:

(i) The Euclidean-normalized differential source counts, determined over a flux range from $10 \mathrm{mJy}$ to $1 \mathrm{Jy}$, are well approximated by a single power-law slope of 0.91 . The inconsistency with model source counts by Jackson (2005) and Wilman et al. (2008) seems due to uncertainty in the predicted contribution of FRI galaxies. Our survey is not deep enough to detect the flattening at the lowest flux densities as seen at higher frequencies (Sect. 4.1).

(ii) The spectral indices of a subset of 417 sources between $153 \mathrm{MHz}$ and $1.4 \mathrm{GHz}$, limited predominantly by the $153 \mathrm{MHz}$ sensitivity, have a median spectral index of -0.76 . We confirm the flattening trend in the median spectral index towards lower $153 \mathrm{MHz}$ flux densities, as seen at higher frequencies (Sect. 4.2). (iii) The detection fraction of USS sources, having a spectral index below -1.3 , is 3.8 percent, which is comparable to the USS fractions of two comparable $153 \mathrm{MHz}$ surveys. Six of the 16 USS sources, apparently physically unrelated, are found in pairs $<6^{\prime}$ apart, which is an unlikely spatial distribution to appear at random (Sect. 4.2).

(iv) The NIR $K_{\mathrm{S}}$-band identification fraction of a subset of 368 sources drops from $\sim 0.7$ for spectral indices $>-0.7$ to $\sim 0.3$ for spectral indices $<-0.7$, reproducing a previously observed correlation (e.g., Wieringa 1991) which links together the known correlations between $K$-band magnitude and redshift, and between spectral index and redshift (Tielens et al. 1979; Blumenthal \& Miley 1979; Willott et al. 2003; Rocca-Volmerange et al. 2004) (Sect. 4.3).

We plan to continue our analysis of the Boötes $153 \mathrm{MHz}$ source survey by comparing it with many other available catalogs at various spectral bands. A high priority task will be to study the properties of the 16 USS sources, to determine if these objects are HzRGs, derive estimates of their redshifts and search the surrounding area for galaxies at similar redshift. This approach has been succesful for the identification and study of galaxy cluster formation (e.g., Röttgering et al. 1994; Venemans et al. 2002).

The observations presented here are part of a larger survey with six additional, partly overlapping flanking fields observed with GMRT at the same frequency, covering a total survey area of $\sim 40$ square degrees. This same area is also covered by extended WSRT observations using 8 bands between 115$165 \mathrm{MHz}$. We will combine these observations with the observations presented here to produce a combined high- and lowresolution catalog at $\sim 153 \mathrm{MHz}$ to further facilitate the study of the low-frequency sky, and in particular to facilitate the further search for USS radio sources.

Acknowledgements. The authors thank Jim Condon, Ed Fomalont and Bill Cotton for useful discussions, and the anonymous referee for providing useful comments. We would also like to thank the staff of the GMRT that made these observations possible. GMRT is run by the National Centre for Radio Astrophysics of the Tata Institute of Fundamental Research. This study made use of online available maps and catalogs from the WSRT Boötes Deep Field survey (de Vries et al. 2002) and the FLAMINGOS Extragalactic Survey (Elston et al. 2006). We are grateful to Niruj Mohan for providing support for the source extraction tool BDSM. H.T.I. acknowledges a grant from the Netherlands Research School for Astronomy (NOVA). This work was partly supported by funding associated with an Academy Professorship of the Royal Netherlands Academy of Arts and Sciences (KNAW), and by the National Radio Astronomy Observatory, which is operated by Associated Universities, Inc., under cooperative agreement with the National Science Foundation.

\section{References}

Afonso, J., Bizzocchi, L., Ibar, E., et al. 2011, ApJ, accepted [arXiv: 1108.4037]

Athreya, R. 2009, ApJ, 696, 885

Baars, J. W. M., Genzel, R., Pauliny-Toth, I. I. K., \& Witzel, A. 1977, A\&A, 61, 99

Becker, R. H., White, R. L., \& Helfand, D. J. 1995, ApJ, 450, 559

Bennett, A. 1962, MmRAS, 68, 163

Bhatnagar, S., Cornwell, T. J., Golap, K., \& Uson, J. M. 2008, A\&A, 487, 419

Blumenthal, G., \& Miley, G. 1979, A\&A, 80, 13

Blundell, K. M., Rawlings, S., \& Willott, C. J. 1999, AJ, 117, 677

Bondi, M., Ciliegi, P., Venturi, T., et al. 2007, A\&A, 463, 519

Bornancini, C. G., O’Mill, A. L., Gurovich, S., \& Lambas, D. G. 2010, MNRAS, 406, 197

Braude, S. Y., Rashkovsky, S. L., Sidorchuk, K. M., et al. 2002, Ap\&SS, 280, 235

Bridle, A., \& Greisen, E. 1994, The NRAO AIPS Project - A Summary, AIPS Memo 87, Tech. Rep., NRAO

Briggs, D. 1995, Ph.D. Thesis, New Mexico Institute of Mining Technology, Socorro, New Mexico, USA

Chandra, P., Ray, A., \& Bhatnagar, S. 2004, ApJ, 612, 974 
Ciliegi, P., Zamorani, G., Hasinger, G., et al. 2003, A\&A, 398, 901

Cohen, A. S., Röttgering, H. J. A., Jarvis, M. J., Kassim, N. E., \& Lazio, T. J. W. 2004, ApJS, 150, 417

Cohen, A. S., Lane, W. M., Cotton, W. D., et al. 2007, AJ, 134, 1245

Condon, J. J. 1997, PASP, 109, 166

Condon, J. J., Cotton, W. D., Greisen, E. W., et al. 1994, in ADASS III, ed. D. R. Crabtree, R. J. Hanisch, \& J. Barnes, ASPC Ser., 61, 155

Condon, J. J., Cotton, W. D., Greisen, E. W., et al. 1998, AJ, 115, 1693

Conway, J. E., Cornwell, T. J., \& Wilkinson, P. N. 1990, MNRAS, 246, 490

Cool, R. J. 2007, ApJS, 169, 21

Cool, R. J., Kochanek, C. S., Eisenstein, D. J., et al. 2006, AJ, 132, 823

Cornwell, T. J., \& Perley, R. A. 1992, A\&A, 261, 353

Cornwell, T., Braun, R., \& Briggs, D. S. 1999, in Synthesis Imaging in Radio Astronomy II, ed. G. B. Taylor, C. L. Carilli, \& R. A. Perley, ASPC Ser., 180, 151

Cotton, W. D. 1999, in Synthesis Imaging in Radio Astronomy II, ed. G. B. Taylor, C. L. Carilli, \& R. A. Perley, ASPC Ser., 180, 357

Croft, S., van Breugel, W., Brown, M. J. I., et al. 2008, AJ, 135, 1793

De Breuck, C., van Breugel, W., Stanford, S. A., et al. 2002, AJ, 123, 637

de Vries, W. H., Morganti, R., Röttgering, H. J. A., et al. 2002, AJ, 123, 1784

Edge, D. O., Shakeshaft, J. R., McAdam, W. B., Baldwin, J. E., \& Archer, S. 1959, MmRAS, 68, 37

Eisenhardt, P. R., Stern, D., Brodwin, M., et al. 2004, ApJS, 154, 48

Elston, R. J., Gonzalez, A. H., McKenzie, E., et al. 2006, ApJ, 639, 816

Feretti, L., \& Johnston-Hollitt, M. 2004, New Astron. Rev., 48, 1145

George, S. J., \& Ishwara-Chandra, C. H. 2009, in The Low-Frequency Radio Universe, ed. D. J. Saikia, D. A. Green, Y. Gupta, \& T. Venturi, ASPC Ser., 407, 47

George, S. J., \& Stevens, I. R. 2008, MNRAS, 390, 741

Giovannini, G., \& Feretti, L. 2000, New Astron., 5, 335

Gower, J. F. R., Scott, P. F., \& Wills, D. 1967, MmRAS, 71, 49

Hales, S. E. G., Baldwin, J. E., \& Warner, P. J. 1988, MNRAS, 234, 919

Hales, S. E. G., Riley, J. M., Waldram, E. M., Warner, P. J., \& Baldwin, J. E. 2007, MNRAS, 382, 1639

Hanisch, R. J. 1982, A\&A, 116, 137

Haslam, C. G. T., Salter, C. J., Stoffel, H., \& Wilson, W. E. 1982, A\&AS, 47, 1

Houck, J. R., Soifer, B. T., Weedman, D., et al. 2005, ApJ, 622, L105

Intema, H. T., van der Tol, S., Cotton, W. D., et al. 2009, A\&A, 501, 1185

Ishwara-Chandra, C. H., \& Marathe, R. 2007, in Deepest Astronomical Surveys,

ed. J. Afonso, H. C. Ferguson, B. Mobasher, \& R. Norris, ASPC Ser., 380, 237

Ishwara-Chandra, C. H., Sirothia, S. K., Wadadekar, Y., Pal, S., \& Windhorst, R. 2010, MNRAS, 405, 436

Jackson, C. 2005, PASA, 22, 36

Jacobson, A. R., \& Erickson, W. C. 1992, A\&A, 257, 401

Jannuzi, B. T., \& Dey, A. 1999, in Photometric Redshifts and the Detection of High Redshift Galaxies, ed. R. Weymann, L. Storrie-Lombardi, M. Sawicki, \& R. Brunner, ASPC Ser., 191, 111

Kempner, J. C., \& Sarazin, C. L. 2001, ApJ, 548, 639
Kenter, A., Murray, S. S., Forman, W. R., et al. 2005, ApJS, 161, 9

Klamer, I. J., Ekers, R. D., Bryant, J. J., et al. 2006, MNRAS, 371, 852

Komatsu, E., Smith, K. M., Dunkley, J., et al. 2011, ApJS, 192, 18

Laing, R. A., Riley, J. M., \& Longair, M. S. 1983, MNRAS, 204, 151

McGilchrist, M. M., Baldwin, J. E., Riley, J. M., et al. 1990, MNRAS, 246, 110

McGreer, I. D., Becker, R. H., Helfand, D. J., \& White, R. L. 2006, ApJ, 652, 157

Miley, G. 1980, ARA\&A, 18, 165

Miley, G., \& De Breuck, C. 2008, A\&AR, 15, 67

Mohan, N. R. 2008, ANAAMIKA manual - version 2.1, Tech. Rep., Leiden Observatory, http://www.strw.leidenuniv.nl/ mohan/anaamika_ manual.pdf

Murray, S. S., Kenter, A., Forman, W. R., et al. 2005, ApJS, 161, 1

Nityananda, R. 2003, in The Proceedings of the IAU 8th Asian-Pacific Regional Meeting, Vol. I, ed. S. Ikeuchi, J. Hearnshaw, \& T. Hanawa, ASPC Ser., 289, 29

Owen, F. N., Morrison, G. E., Klimek, M. D., \& Greisen, E. W. 2009, AJ, 137, 4846

Pandey, V. N., \& Shankar, N. U. 2007, Highlights of Astronomy, 14, 385

Perley, R. A. 1989, in Synthesis Imaging in Radio Astronomy, ed. R. A. Perley, F. R. Schwab, \& A. H. Bridle, ASPC Ser., 6, 287

Pilkington, J. D. H., \& Scott, P. F. 1965, MmRAS, 69, 183

Prandoni, I., Parma, P., Wieringa, M. H., et al. 2006, A\&A, 457, 517

Rengelink, R. B., Tang, Y., de Bruyn, A. G., et al. 1997, A\&AS, 124, 259

Rocca-Volmerange, B., Le Borgne, D., De Breuck, C., Fioc, M., \& Moy, E. 2004, A\&A, 415, 931

Röttgering, H. J. A., van Ojik, R., Miley, G. K., et al. 1997, A\&A, 326, 505

Röttgering, H. J. A., Lacy, M., Miley, G. K., Chambers, K. C., \& Saunders, R. 1994, A\&AS, 108, 79

Schwab, F. R. 1984, AJ, 89, 1076

Sirothia, S. K., Saikia, D. J., Ishwara-Chandra, C. H., \& Kantharia, N. G. 2009, MNRAS, 392, 1403

Sutherland, W., \& Saunders, W. 1992, MNRAS, 259, 413

Tasse, C., Cohen, A. S., Röttgering, H. J. A., et al. 2006, A\&A, 456, 791

Tasse, C., Röttgering, H. J. A., Best, P. N., et al. 2007, A\&A, 471, 1105

Tasse, C., Le Borgne, D., Röttgering, H., et al. 2008, A\&A, 490, 879

Thompson, A. R. 1999, in Synthesis Imaging in Radio Astronomy II, ed. G. B. Taylor, C. L. Carilli, \& R. A. Perley, ASPC Ser., 180, 11

Tielens, A. G. G. M., Miley, G. K., \& Willis, A. G. 1979, A\&AS, 35, 153

van Breugel, W., De Breuck, C., Stanford, S. A., et al. 1999, ApJ, 518, L61

Venemans, B. P., Kurk, J. D., Miley, G. K., et al. 2002, ApJ, 569, L11

Venemans, B. P., Röttgering, H. J. A., Miley, G. K., et al. 2007, A\&A, 461, 823

Wieringa, M. H. 1991, Ph.D. Thesis, Leiden Observatory, Leiden University, Leiden, The Netherlands

Willott, C. J., Rawlings, S., Jarvis, M. J., \& Blundell, K. M. 2003, MNRAS, 339, 173

Wilman, R. J., Miller, L., Jarvis, M. J., et al. 2008, MNRAS, 388, 1335

Zhang, X.-Z., Reich, W., Reich, P., \& Wielebinski, R. 2003, Chin. J. Astron. Astrophys., 3, 347 
H. T. Intema et al.: Deep low-frequency radio observations of the Boötes field. I.

\section{Appendix A: Selection of $153 \mathrm{MHz}$ radio source images}
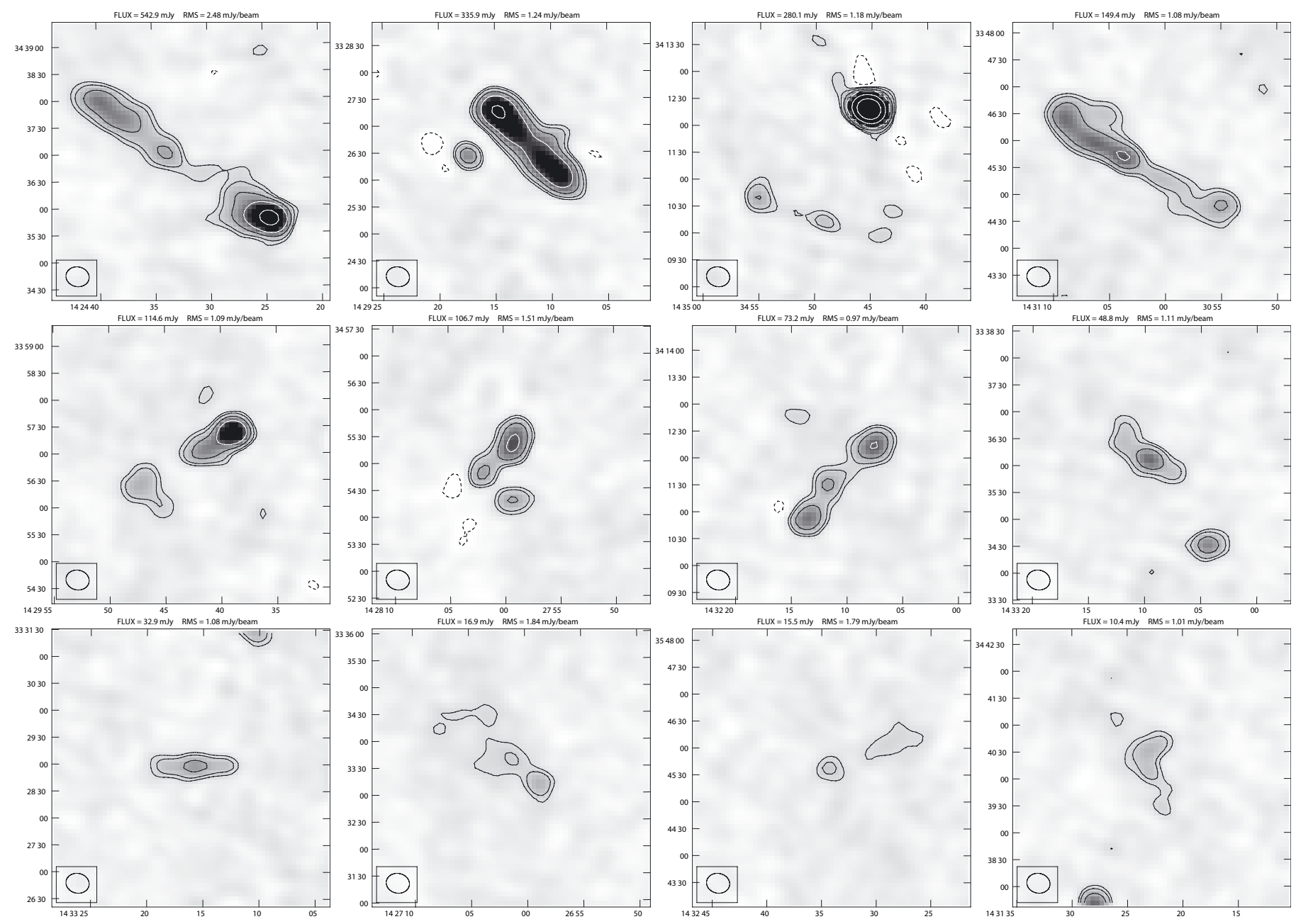

Fig. A.1. Grey-scale images of a selection of resolved $153 \mathrm{MHz}$ sources with a complex morphology (i.e., not a point source or double point source morphology). Horizontal and vertical axes are RA and Dec, respectively. For each source, the total flux and local rms noise are specified above the $5^{\prime} \times 5^{\prime}$ image. Contours are drawn at $[-3,3,5,10,20,50,100]$ times the local noise. The grey-scale ranges from -3 to 30 times the local noise. 